\title{
Addiction

\section{A systematic review of studies assessing the association between adherence to smoking cessation medication and treatment success}

\begin{tabular}{|c|c|}
\hline Journal: & Addiction \\
\hline Manuscript ID: & ADD-13-0373.R2 \\
\hline Manuscript Type: & Review \\
\hline Date Submitted by the Author: & $\mathrm{n} / \mathrm{a}$ \\
\hline Complete List of Authors: & $\begin{array}{l}\text { Raupach, Tobias; University Hospital Goettingen, Cardiology and } \\
\text { Pneumology } \\
\text { Brown, Jamie; University College London, Health Behaviour Research } \\
\text { Centre } \\
\text { Herbec, Aleksandra; University College London, Health Behaviour Research } \\
\text { Centre } \\
\text { Brose, Leonie; University College London, National Centre for Smoking } \\
\text { Cessation and Training } \\
\text { West, Robert; University College London, Health Behaviour Research } \\
\text { Centre }\end{array}$ \\
\hline SUBSTANCE: & tobacco \\
\hline METHOD: & Review \\
\hline FIELD OF STUDY: & medicine \\
\hline Keywords: & $\begin{array}{l}\text { adherence, cessation, compliance, medication, smoking, success, quitting, } \\
\text { nicotine replacement therapy, bupropion, varenicline }\end{array}$ \\
\hline
\end{tabular}




\title{
A systematic review of studies assessing the association between adherence to smoking cessation medication and treatment success
}

\author{
Tobias Raupach ${ }^{1,2}$ \\ Jamie Brown ${ }^{2}$ \\ Aleksandra Herbec ${ }^{2}$ \\ Leonie Brose ${ }^{3}$ \\ Robert West ${ }^{2}$
}

\begin{abstract}
Affiliations
${ }^{1}$ Department of Cardiology and Pneumology, University Hospital Göttingen Göttingen, Germany

${ }^{2}$ Cancer Research UK Health Behaviour Research Centre, University College London, London, UK

${ }^{3}$ National Centre for Smoking Cessation and Training (NCSCT), University College London, London, UK
\end{abstract}

Running head

Word count text body

Correspondence:
Adherence to pharmacotherapy for smoking cessation

3767

Tobias Raupach, MD

University Hospital Göttingen

Department of Cardiology and Pneumology

Robert-Koch-Straße 40

D-37075 Göttingen, Germany

Phone: +49 551 39-8922

Fax: +49 551 39-6887

E-mail: raupach@med.uni-goettingen.de 


\section{Abstract}

Aims: Lack of adherence to smoking cessation medication regimens is assumed to play a significant role in limiting their effectiveness. This study aimed to assess evidence for this assumption.

Methods: A systematic search was conducted, supplemented by expert consultation, of articles reporting on randomised trials and observational studies examining the association between adherence to cessation medication and the success of quit attempts. To rule out reverse causality, only studies where adherence was assessed prior to relapse were included. Five studies met the inclusion criteria and results were extracted independently by two researchers. Heterogeneity between studies precluded a pooled analysis of the data.

Results: Studies varied widely with regard to both the definition of adherence and outcome measures. Included studies only addressed adherence to nicotine replacement therapy. One study of lozenge use found that amount of medication used between 1 and 2 weeks after the quit date predicted abstinence at 6 weeks (adjusted OR for 'high' versus 'low' lozenge use $1.25 ; 95 \%$ confidence interval $(\mathrm{Cl})=1.05-1.50 ; \mathrm{p}<0.02)$. Similarly, one study found a significant impact of oral nicotine consumption during the first week on abstinence at four weeks (adjusted OR per additional $\mathrm{mg} / \mathrm{d}: 1.05 \% ; \mathrm{Cl}=1.01-1.10$ ). Another study found that participants using nicotine replacement therapy for at least five weeks were significantly more likely to self-report continuous abstinence at 6 months. The remaining two studies failed to find a significant effect of treatment duration on outcome at one and two years but had very low power to detect such an effect.

Conclusions: There is modest evidence to support the assumption that lack of adherence to nicotine replacement therapy regimens undermines effectiveness in clinical studies.

Key words: adherence, cessation, compliance, medication, smoking, success, quitting, nicotine replacement therapy, bupropion, varenicline 


\section{Introduction}

Data from numerous randomised controlled trials clearly demonstrate the effectiveness of nicotine replacement therapy (NRT) (1), bupropion (2) and varenicline (3) in promoting longterm abstinence from smoking. However, some population studies suggest that pharmacotherapy may be considerably less effective outside clinical trials (4). One possible explanation for the finding of lower effectiveness in the 'real world' is that many smokers fail to adhere to treatment recommendations, i.e. they tend to take inadequate doses $(5,6)$ or discontinue treatment early (7). The amount of medication taken is likely to have a moderating effect on the effectiveness of drugs used to assist quit attempts. In randomised controlled trials, great care is being taken to ensure good patient adherence. Nevertheless, a substantial proportion of study participants do not appear to follow dosing instructions: In one early trial on nicotine gum in which patients were advised to use their medication for at least three months, $43 \%$ of participants in the active treatment arm stopped taking the gum within 4 weeks (8). Similarly high rates of early discontinuation have been reported for the nicotine patch (9), bupropion (10) and varenicline (11). There is evidence to suggest that adherence to cessation medication is even lower outside clinical trials: In one retrospective survey from the United States, past NRT users who had bought their medication over the counter reported a median treatment duration of 9.8 days (12) which is in contrast with manufacturer recommendations (at least 8 weeks). In one prospective study from China, $84 \%$ of participants used NRT for less than 4 weeks, and $44 \%$ used it for less than 7 days (13).

There is currently no consensus on what defines adequate adherence in the context of smoking cessation medication. Adherence can be defined as compliance with recommendations on treatment duration or as compliance with a given dosing regimen. A general definition of good adherence to oral medication for the treatment of chronic diseases is use for at least $80 \%$ of the recommended duration (14). Due to the diversity regarding the route of administration of medications to support smoking cessation (i.e., nasal, dermal or oral application), a universal criterion for adherence to these drugs is particularly hard to define. As a consequence, studies addressing adherence have used a wide range of definitions, e.g. 'taking at least 1 dose of medication for at least $80 \%$ of the treatment days' (15), 'chewing at least 10 pieces of nicotine gum per day' (16), and compliance indices calculated as the proportion of scheduled doses that had actually been taken $(17,18)$.

Some of the reasons for early termination of cessation medication quoted most frequently in surveys include adverse events $(12,15,19-21)$, medication cost $(12,21)$ and no perceived need to take medication to stop smoking $(12,19,20)$. The most important precipitating factor for medication non-adherence, however, is likely to be relapse to smoking. In a recent inter- 
net survey on the use of various medications to support a quit attempt (21), $42 \%$ of participants stated they had stopped using the nicotine patch because they had relapsed to smoking; the corresponding proportions for other medications were $52 \%$ (nicotine gum), $46 \%$ (nicotine lozenge/tablet), 54\% (nicotine inhaler), 26\% (bupropion), and 18\% (varenicline). Studies assessing the association between adherence to medication and success of a quit attempt might not yield valid results if non-adherence was not the cause but the consequence of relapse in a substantial proportion of cases. This effect which has also been termed 'reverse causality' (22) is likely to lead to an overestimation of the effect of treatment duration on quitting success as more treatment failures with short durations of treatment would be included in the analysis. This review aims to summarise the available evidence on the association between adherence and abstinence in studies controlling for potential bias due to relapse precipitating discontinuation of medication use.

\section{Methods}

\section{Search strategy}

Online databases (PubMed, WebOfScience, and the Cochrane Tobacco Addiction Group specialized register) were searched up to 28 February 2013 with the terms: 'smoking cessation AND (adherence OR compliance) AND (abstinence OR success)'. An additional search included the terms: '(nicotine replacement OR bupropion OR varenicline) AND (adherence OR compliance)'. Search terms were inclusive in an attempt to locate all studies examining the association between adherence and abstinence. A hand-search of the reference lists of included studies was also carried out, and leading researchers in the field were contacted. Studies identified by these searches were screened for eligibility by two reviewers (T.R. and A.H.), with $98.8 \%$ agreement. In six cases, consensus was reached by involving a third reviewer (J.B.) who was blinded to the other reviewers' assessments. Details of the method of data collection, outcome measures, recall period, participant characteristics, sample size, response rate and analysis method were extracted and compiled into a table independently by two researchers (T.R. and A.H). All discrepancies were checked against the study papers, discussed and resolved.

\section{Inclusion and exclusion criteria}

We included primary and secondary analyses of prospective randomised controlled trials and observational studies which specifically addressed the association between medication adherence and abstinence in adult smokers. Due to potential confounding by recall bias, purely retrospective surveys were not included. With regard to pharmacotherapies, only studies 
involving the use of nicotine replacement therapy, buproprion or varenicline (used alone or in combination) were included as these are considered first-line treatments in most countries $(23,24)$. We only included original articles written in English and published in peer-reviewed journals. Review articles, personal communications to editors, commentaries, study protocols, case studies, studies on smoking reduction and studies involving pregnant women or adolescents were excluded.

As outlined above, an important potential confounder in studies assessing the association between treatment adherence and abstinence is relapse leading to non-adherence in which case non-adherence is not the cause but the consequence of relapse. There are two ways to control for this bias:

a) establishing the chronological sequence of non-adherence and relapse during a study

b) assessing adherence during a pre-specified treatment period and determine abstinence only in those who had been continuously abstinent throughout this period

Only studies reporting a valid strategy to control for reverse causality were included in this review.

\section{Outcome measures}

There was no uniform definition of adherence; most studies used retrospective self-reports of drug use to assess adherence while some interviewed participants daily via an interactive voice response system or established adherence using medication dispensers with an electronic counting device fitted to the bottle cap. Details of the definitions and methods used in individual studies are given in Table 1 and Table S1 (online supplement of this article).

Abstinence was defined as the proportion of participants who achieved point prevalence, 7day point prevalence or continuous abstinence up to a given time-point. The assessment of abstinence was based on self-report or biochemical validation by exhaled carbon monoxide or salivary cotinine concentrations, and different cut-off values were used in different studies.

\section{Data analysis}

Due to variation between the studies with regard to the definitions of adherence and abstinence, results could not be pooled statistically. Consequently, the evidence was synthesized in a narrative review. 


\section{Results}

\section{Search results}

The electronic literature search yielded 498 articles. For 119 of these, eligibility could not be determined from the abstract so full text versions were retrieved and studied in detail. Thirty further eligible articles were identified through a review of reference lists and one additional article through contacting experts in the field. Of the resulting 150 articles, 37 assessed the association between adherence and abstinence, but only five reported using a strategy to control for potential confounding by reverse causality and were thus included in this review. The authors of one additional study (25) took a different approach to controlling for such confounding in that they adjusted for smoking status during the first three weeks of a trial in a logistic regression of predictors of abstinence at six weeks. In this regard, that study did not meet the inclusion criteria for this review; however, its findings were similar to the results of a study from the same group that was included in this review (22).

\section{Description of included studies}

All five articles assessed the association between NRT use and abstinence; this research aim was explicitly stated in three studies $(22,26,27)$ and addressed in sub-group analyses in the other two $(28,29)$. Two articles presented secondary analyses of randomised controlled trials $(22,27)$, and two articles provided data from prospective observational studies $(26,28)$. The only article reporting original results of a randomised controlled trial referred to a study of nicotine gum versus placebo in addition to nicotine patch treatment in a small sample $(\mathrm{n}=$ 96) of alcohol-dependent smokers in an early phase of out-patient alcohol treatment (29).

One study was conducted in the United Kingdom (27), one in the United States (29), one enrolled patients in both countries (22), and the two remaining studies were from Switzerland (26) and Germany (28), respectively. Baseline sample sizes ranged from 92 to 1,030, study populations were predominantly white, the mean/median age of participants ranged from 40 to 47 years, $29 \%$ to $54 \%$ of participants were female, and the mean/median number of cigarettes smoked daily ranged from 20 to 25 . The length of follow-up ranged from four weeks to two years. Each study took a different approach to measuring adherence (see below). Smoking outcome was assessed as continuous abstinence and validated by exhaled carbon monoxide (CO) in four of the five studies $(22,26,27,29)$. The association between adherence and abstinence was assessed by means of a logistic regression in four and by a $\chi^{2}$ test in one study (28). 
Details of the 37 articles addressing the association between adherence and abstinence but not controlling for relapse as a cause for non-adherence are provided in Table S1 in the online supplement to this article. Information on included studies is summarised in Table 1.

\section{Summary of the evidence}

Due to the heterogeneity of the studies discussed above, this section provides short narrative summaries of the five included studies.

1. Shiffman (22) conducted a secondary analysis of a randomised controlled trial of nicotine lozenges versus placebo in 1,030 smokers. Participants were instructed to use lozenges for 6 weeks. Adherence to study medication was monitored daily during the first two weeks of the trial, using an interactive voice response system. In the absence of an a priori definition of adherence, study participants were categorised as 'high' lozenge users or 'low' lozenge users based on a median split of the entire cohort. The mean number of lozenges used per day was $10.2 \pm 2.5$ in the 'high' users group and $5.1 \pm 1.9$ in the 'low' users group. Smoking outcome was defined as continuous 28-day abstinence, validated by exhaled $\mathrm{CO}$ at 6 weeks. In order to control for confounding by non-adherence due to relapse, the analysis (logistic regression) only included participants that had remained abstinent for the first two weeks of the trial (i.e. the period during which adherence was monitored daily). Thus, a dichotomised parameter of lozenge use during the first two weeks was examined as a predictor of continuous abstinence at six weeks in those who had been randomised to active treatment and who had not relapsed during the first two weeks (sample size not reported). The odds of continuous abstinence were significantly higher for 'high' lozenge users in both the unadjusted model (OR 1.60; 95\% confidence interval $(\mathrm{Cl})$ : 1.13-2.27; $\mathrm{p}<0.009)$ and a model adjusting for gender and numbers of cigarettes smoked at study entry (OR 1.25; $\mathrm{Cl}=1.05-1.50 ; \mathrm{p}<0.02)$. When entered as a continuous variable, each additional lozenge per day significantly increased the odds of achieving abstinence by $10 \%(4-16 \%)$ in both the unadjusted and the adjusted model.

2. Hollands et al. (27) report the results of a secondary analysis of data from a randomised controlled trial in a primary care setting. All participants received a nicotine patch (dose tailored to the number of cigarettes smoked per day) and additional oral NRT. Participants were randomised to have their oral dose calculated based on (a) their genotype (presence/absence of a specific mutation; see (30) for details) or (b) their level of nicotine dependence as measured by the Fagerström Test of Nicotine Dependence (FTND (31)). Adherence during the first trial week was operationalised as NRT consumption and measured in $\mathrm{mg} / \mathrm{d}$. Smoking outcome was defined as 4-week abstinence, validated by exhaled $\mathrm{CO}$. In order to control for confounding by non-adherence due to relapse, the 
analysis (logistic regression) only included participants that had remained abstinent for the first trial week. Thus, a continuous measure of NRT use during the first week was examined as a predictor of continuous abstinence at four weeks in those who had not relapsed during the first week $(n=285)$. The odds of abstinence increased by $5 \%(\mathrm{Cl}=1$ $10 \%$ ) per additional $\mathrm{mg} / \mathrm{d}$ in a model adjusting for various confounders, including nicotine dependence and treatment arm of the randomised trial.

3. Cooney et al. (29) randomised 96 alcohol-dependent smokers in an early phase of outpatient alcohol treatment (two study sites) to nicotine gum versus placebo on top of a 12week course of nicotine patches. Participants were encouraged to use between 6 and 20 pieces of gum per day. There was no a priori definition of adherence; medication use was assessed two weeks after the target quit date by eliciting a 7-day retrospective report of gum use during patient interviews. The frequency of gum use at two weeks was entered into logistic regressions of predictors of continuous abstinence (validated by exhaled $\mathrm{CO}$ ) at 3, 6 and 12 months. In order to control for confounding by non-adherence due to relapse, the final analysis only included participants that had remained abstinent during the first two weeks ( $n=37$ ). After adjusting for educational level, depression score, nicotine dependence and study site, more frequent use of study medication (gum or placebo) during the second week of the first two treatment weeks increased the odds of continuous abstinence at 3,6 and 12 months by $4 \%(\mathrm{Cl}=1 \%$ to $6 \% ; \mathrm{p}=0.008), 4 \%(1 \%$ to $8 \% ; \mathrm{p}=$ $0.045)$ and $3 \%(-3 \%$ to $10 \% ; p=0.364)$, respectively.

4. Raupach et al. (28) followed up 369 participants of a hospital-based smoking cessation programme for 6 months who had been encouraged to purchase NRT themselves. Participants provided self-reports of continuous abstinence and treatment duration at the sixmonth telephone follow-up. In the absence of an a priori definition of adherence, this study considered a minimum treatment duration of five weeks to indicate good adherence. In order to control for confounding by non-adherence due to relapse, analysis of the association between adherence and abstinence was restricted to those who had either remained abstinent or relapsed only after discontinuing medication use $(n=127)$. Within this sub-group, self-reported continuous abstinence rate at 6 months was significantly higher if medication had been used for at least five weeks $(61.0 \%$ vs. $42.6 \%$; $p=$ 0.039).

5. Schneider et al. (26) followed up 92 smokers who were provided with nicotine nasal spray to be used ad libitum for up to 18 months. During the first month of the study, spray use was monitored using a metered-dose inhaler fitted with an electronic device recording the date and time of each use. There was no a priori definition of adherence, and continuous abstinence from the end of the first month was assessed and validated by ex- 
haled $\mathrm{CO}$ at a clinic visit two years after study entry. The methods report that in order to control for confounding by non-adherence due to relapse, only participants who had remained abstinent during the first month $(n=48)$ were included in the final analysis. In the multiple regression, median daily consumption of nasal spray was not predictive of continuous abstinence at 2 years (no ORs provided). It should be noted that the reporting in the results was brief and without exact figures, which meant there was no numerical confirmation of the stated methods that the analysis would be limited to the appropriate subgroup.

\section{Discussion}

\section{Main findings of this review}

The results of this review indicate that there is a substantial lack of high-quality studies assessing the association between treatment adherence and subsequent quitting success. The two studies with the most rigorous control for confounding by reverse causality $(22,27)$ both found a significant effect of the amount of medication taken and quit rates at four to six weeks. The only other study reporting a significant effect on continuous 6-month abstinence (28) was limited by its observational design, a lack of biochemical validation of smoking status and potential confounding by participant motivation and recall bias. The two remaining studies which did not find significant effects after one (29) and two (26) years appeared underpowered as sample sizes were small. Since all five studies that met our inclusion criteria addressed adherence to NRT products, no conclusions can currently be drawn on the association between adherence and treatment success for other first-line treatments such as bupropion and varenicline, or combinations of treatments.

\section{Strengths and limitations}

In order to ensure the inclusion of all relevant articles, two independent reviewers assessed all publications identified by an extensive search of the literature. Agreement between reviewers was high, and all discrepancies were resolved by involving a third independent reviewer. We used conservative inclusion criteria in order to restrict this review to studies with relatively rigid methodology. This led to the exclusion of one study (25) that did not control for reverse causality in the way set out in our criteria but produced similar results as a comparable study with a larger sample size.

Only original articles written in English were included in this review. A total of 25 Pubmed citations were excluded due to their being written in Spanish $(n=9)$, German $(n=8)$, Polish 
$(n=3)$, French $(n=2)$, Dutch $(n=1)$, Turkish $(n=1)$, or Japanese $(n=1)$. Six of these were review articles and had to be excluded for that reason, and one was a commentary. The abstracts of the remaining 18 articles were screened, and none of these assessed abstinence in relation to medication adherence. Thus, exclusion of articles not written in English is unlikely to have confounded our results.

Another limitation of this review is that we were unable to conduct quantitative quality assessments of the included studies. This was due to the fact that there are currently no universally accepted quality criteria for the type of studies included in this review; available tools to assess the quality of such studies have been criticised for their low reliability $(32,33)$. Instead, we used our field-specific expertise to provide qualitative judgments on the quality of included studies. Only two of the five included studies reported results from randomisedcontrolled trials; however, these were derived from secondary analyses. Thus, the association between adherence and abstinence was not a primary endpoint of these studies. The remaining three studies enrolled specific patient groups (i.e., alcohol-dependent smokers or smokers highly motivated to quit who reported to a university-based cessation clinic) which limits the generalisability of their findings to a general smoker population. Sample size was below 100 in two studies, and drop-out rates approached $50 \%$ in one study. Finally, four of the five studies did not use an a priori definition of adherence. In summary, the quality of included studies was low to moderate, and more well-designed studies are clearly needed.

Interpretation of the available evidence is further hampered by the lack of a universal definition of adherence and a consensus on how to control for reverse causality. Recently, it has been suggested to report adherence as the percentage of prescribed amount or to directly calculate medication intake (27). Excluding participants who stopped using NRT because they abandoned their quit attempt (28) would be desirable but can only be done if all relevant data are available. The alternative approach taken by some authors (i.e. relating adherence during a short interval at the beginning of a trial to abstinence at a later stage) is more problematic as it does not account for (non-)adherence between the initial adherence period and the time when the quit attempt ended. While one study on medium-term abstinence retrospectively assessed adherence throughout the entire treatment phase (28), the two other small studies assessing abstinence at one (29) or two years (26) only controlled for reverse causality during the first 2-4 weeks of the treatment phase. Thus, even in these studies, a residual bias arising from reverse causality cannot be excluded.

\section{Suggestions for future research}

The definitions of adherence used in these studies were not primarily based on theoretical considerations including the mode of action of pharmacotherapies but mainly derived post 
hoc from the data (e.g., median split of the number of lozenges taken per day or an arbitrary cut-off of at least 5 weeks of treatment). The fact that relapse tends to occur early during a quit attempt (34) suggests that the first weeks of treatment are most important, but no firm conclusions can be drawn from the available literature. Identification of a minimum treatment duration (or amount of medication taken per day) for pharmacotherapy to be effective is important in order to design interventions that may increase adherence (35-38). Ideally, such interventions would be informed by an analysis of modifiable predictors of adherence.

Despite the lack of a universal (and clinically meaningful) definition of good adherence, a number of studies have reported on predictors of adherence. These studies used various designs including secondary analyses of randomised controlled trial data (19), prospective observations (13) and retrospective surveys (20,39). Factors that were found to be associated with better adherence by most studies included male gender (13), more advanced age $(13,15,19,20)$, higher self-efficacy $(19,40)$, lower smoking rate at study entry (15), and more intensive concomitant counselling (41). However, since non-adherence may be precipitated by relapse in up to $50 \%$ of cases (21), these might reflect characteristics associated with higher odds of successful quit attempts regardless of medication adherence. In fact, most of the predictors listed above have been found to independently increase quit rates in a number of studies (42).

In conclusion, we found some evidence in studies of nicotine replacement therapy that low rates of adherence may be limiting effectiveness in clinical trials. These findings need to be confirmed using more rigorous methods (e.g. by assessing adherence using medication dispenser systems with an electronic monitoring device (37) up to a pre-defined follow-up point or the end of a quit attempt). They also need to be extended to other stop smoking medications and to use of stop smoking medicines outside of clinical studies.

\section{Declarations of interest}

TR has received honoraria from Pfizer ${ }^{\circledR}$, Novartis ${ }^{\circledR}$, Glaxo Smith Kline ${ }^{\circledR}$, Astra Zeneca ${ }^{\circledR}$ and Roche $^{\circledR}$ as a speaker in activities related to continuing medical education and smoking cessation. RW undertakes consultancy and research for and receives travel funds and hospitality from manufacturers of medications for smoking cessation. He also undertakes training for smoking cessation advisors and has a share of a patent for a novel nicotine delivery device. $\mathrm{JB}, \mathrm{AH}$ and $\mathrm{LB}$ have no competing interests. 


\section{References}

1. Stead, L. F., Perera, R., Bullen, C., Mant, D. \& Lancaster, T. (2008) Nicotine replacement therapy for smoking cessation, Cochrane Database Syst Rev, CD000146.

2. Hughes, J. R., STEAd, L. F. \& LAnCAster, T. (2007) Antidepressants for smoking cessation, Cochrane Database Syst Rev, CD000031.

3. CAHILl, K., StEAD, L. F. \& LANCASTER, T. (2012) Nicotine receptor partial agonists for smoking cessation, Cochrane Database Syst Rev, 4, CD006103.

4. Alberg, A. J., Patnaik, J. L., May, J. W. et al. (2005) Nicotine replacement therapy use among a cohort of smokers, $J$ Addict Dis, 24, 101-13.

5. Johnson, R. E., Stevens, V. J., Hollis, J. F. \& Woodson, G. T. (1992) Nicotine chewing gum use in the outpatient care setting, J Fam Pract, 34, 61-5.

6. Shiffman, S., Paty, J. A., Rohay, J. M., Di Marino, M. E. \& Gitchell, J. (2000) The efficacy of computer-tailored smoking cessation material as a supplement to nicotine polacrilex gum therapy, Arch Intern Med, 160, 1675-81.

7. Bansal, M. A., Cummings, K. M., Hyland, A. \& Giovino, G. A. (2004) Stopsmoking medications: who uses them, who misuses them, and who is misinformed about them?, Nicotine Tob Res, 6 Suppl 3, S303-10.

8. Jarvis, M. J., RAW, M., Russell, M. A. \& Feyerabend, C. (1982) Randomised controlled trial of nicotine chewing-gum, Br Med J (Clin Res Ed), 285, 537-40.

9. Tonnesen, P., PaOletti, P., Gustavsson, G. et al. (1999) Higher dosage nicotine patches increase one-year smoking cessation rates: results from the European CEASE trial. Collaborative European Anti-Smoking Evaluation. European Respiratory Society, Eur Respir J, 13, 238-46.

10. Fossati, R., Apolone, G., Negri, E. et al. (2007) A double-blind, placebo-controlled, randomized trial of bupropion for smoking cessation in primary care, Arch Intern Med, 167, 1791-7.

11. GonZales, D., Rennard, S. I., Nides, M. et al. (2006) Varenicline, an alpha4beta2 nicotinic acetylcholine receptor partial agonist, vs sustained-release bupropion and placebo for smoking cessation: a randomized controlled trial, JAMA, 296, 47-55.

12. BURNS, E. K. \& LEVINSON, A. H. (2008) Discontinuation of nicotine replacement therapy among smoking-cessation attempters, Am J Prev Med, 34, 212-5.

13. Lam, T. H., Abdullah, A. S., Chan, S. S. \& Hedley, A. J. (2005) Adherence to nicotine replacement therapy versus quitting smoking among Chinese smokers: a preliminary investigation, Psychopharmacology (Berl), 177, 400-8.

14. Dimatteo, M. R., Giordani, P. J., Lepper, H. S. \& Croghan, T. W. (2002) Patient adherence and medical treatment outcomes: a meta-analysis, Med Care, 40, 794-811.

15. Hays, J. T., Leischow, S. J., Lawrence, D. \& LeE, T. C. (2010) Adherence to treatment for tobacco dependence: association with smoking abstinence and predictors of adherence, Nicotine Tob Res, 12, 574-81.

16. Fagerstrom, K. O. (1984) Effects of nicotine chewing gum and follow-up appointments in physician-based smoking cessation, Prev Med, 13, 517-27.

17. Goldstein, M. G., Niaura, R., Follick, M. J. \& Abrams, D. B. (1989) Effects of behavioral skills training and schedule of nicotine gum administration on smoking cessation, Am J Psychiatry, 146, 56-60.

18. Schmitz, J. M., Stotts, A. L., Mooney, M. E., Delaune, K. A. \& Moeller, G. F. (2007) Bupropion and cognitive-behavioral therapy for smoking cessation in women, Nicotine Tob Res, 9, 699-709.

19. CAtz, S. L., JaCK, L. M., McClure, J. B. et al. (2011) Adherence to varenicline in the COMPASS smoking cessation intervention trial, Nicotine Tob Res, 13, 361-8. 
20. Balmford, J., Borland, R., Hammond, D. \& Cummings, K. M. (2011) Adherence to and reasons for premature discontinuation from stop-smoking medications: data from the ITC Four-Country Survey, Nicotine Tob Res, 13, 94-102.

21. EtTer, J. F. \& SChNeIder, N. G. (2012) An Internet Survey of Use, Opinions and Preferences for Smoking Cessation Medications: Nicotine, Varenicline, and Bupropion, Nicotine Tob Res.

22. SHIFFMAN, S. (2007) Use of more nicotine lozenges leads to better success in quitting smoking, Addiction, 102, 809-14.

23. FIORE, C., JAÉN, C. R., BAKER, T. B. et al. (2008) Treating Tobacco Use and Dependence: 2008 Update. Clinical Practice Guideline. (Rockville, MD, U.S. Department of Health and Human Services. Public Health Service.).

24. RAUPACH, T. \& VAN SCHAYCK, C. P. (2011) Pharmacotherapy for smoking cessation: current advances and research topics, CNS Drugs, 25, 371-82.

25. Shiffman, S., Sweeney, C. T., Ferguson, S. G., Sembower, M. A. \& Gitchell, J. G. (2008) Relationship between adherence to daily nicotine patch use and treatment efficacy: secondary analysis of a 10-week randomized, double-blind, placebocontrolled clinical trial simulating over-the-counter use in adult smokers, Clin Ther, 30, 1852-8.

26. SCHNEIDER, M. P., VAN Melle, G., UldRY, C. et al. (2003) Electronic monitoring of long-term use of the nicotine nasal spray and predictors of success in a smoking cessation program, Nicotine Tob Res, 5, 719-27.

27. Hollands, G. J., Sutton, S., McDermott, M. S., Marteau, T. M. \& Aveyard, P. (2013) Adherence to and Consumption of Nicotine Replacement Therapy and the Relationship With Abstinence Within a Smoking Cessation Trial in Primary Care, Nicotine Tob Res.

28. RAuPACH, T., SHAHAB, L., NeUBERT, K. et al. (2008) Implementing a hospital-based smoking cessation programme: evidence for a learning effect, Patient Educ Couns, 70, 199-204.

29. Cooney, N. L., CoOney, J. L., Perry, B. L. et al. (2009) Smoking cessation during alcohol treatment: a randomized trial of combination nicotine patch plus nicotine gum, Addiction, 104, 1588-96.

30. Marteau, T. M., Aveyard, P., Munafo, M. R. et al. (2012) Effect on adherence to nicotine replacement therapy of informing smokers their dose is determined by their genotype: a randomised controlled trial, PLoS One, 7, e35249.

31. Heatherton, T. F., Kozlowski, L. T., Frecker, R. C. \& Fagerstrom, K. O. (1991) The Fagerstrom Test for Nicotine Dependence: a revision of the Fagerstrom Tolerance Questionnaire, Br J Addict, 86, 1119-27.

32. Hartling, L., Milne, A., Hamm, M. P. et al. (2013) Testing the Newcastle Ottawa Scale showed low reliability between individual reviewers, J Clin Epidemiol; in press.

33. Oremus, M., Oremus, C., Hall, G. B. \& McKinnon, M. C. (2012) Inter-rater and test-retest reliability of quality assessments by novice student raters using the Jadad and Newcastle-Ottawa Scales, BMJ Open, 2; e001368.

34. Hughes, J. R., KeEly, J. \& NAud, S. (2004) Shape of the relapse curve and long-term abstinence among untreated smokers, Addiction, 99, 29-38.

35. RAUPACH, T., SHAHAB, L., EIMER, S. et al. (2010) Increasing the use of nicotine replacement therapy by a simple intervention: an exploratory trial, Subst Use Misuse, 45, 403-13.

36. Mooney, M. E., Sayre, S. L., Hokanson, P. S., Stotts, A. L. \& Schmitz, J. M. (2007) Adding MEMS feedback to behavioral smoking cessation therapy increases compliance with bupropion: a replication and extension study, Addict Behav, 32, 87580 . 
37. Schmitz, J. M., Sayre, S. L., Stotts, A. L., Rothfleisch, J. \& Mooney, M. E. (2005) Medication compliance during a smoking cessation clinical trial: a brief intervention using MEMS feedback, J Behav Med, 28, 139-47.

38. Ferguson, S. G., Gitchell, J. G., Shiffman, S. et al. (2011) Providing accurate safety information may increase a smoker's willingness to use nicotine replacement therapy as part of a quit attempt, Addict Behav, 36, 713-6.

39. Shiffman, S., Ferguson, S. G., Rohay, J. \& Gitchell, J. G. (2008) Perceived safety and efficacy of nicotine replacement therapies among US smokers and ex-smokers: relationship with use and compliance, Addiction, 103, 1371-8.

40. Fucito, L. M., Toll, B. A., SAlovey, P. \& O'Malley, S. S. (2009) Beliefs and attitudes about bupropion: implications for medication adherence and smoking cessation treatment, Psychol Addict Behav, 23, 373-9.

41. ORLEAns, C. T., ResCH, N., Noll, E. et al. (1994) Use of transdermal nicotine in a state-level prescription plan for the elderly. A first look at 'real-world' patch users, JAMA, 271, 601-7.

42. Vangeli, E., Stapleton, J., Smit, E. S., Borland, R. \& West, R. (2011) Predictors of attempts to stop smoking and their success in adult general population samples: a systematic review, Addiction, 106, 2110-21. 


\section{Tables}

\begin{tabular}{|c|c|c|c|c|c|c|c|c|c|c|c|}
\hline \multirow{2}{*}{ ID } & \multirow{2}{*}{$\begin{array}{l}\text { Country, Year } \\
\text { and Study } \\
\text { Population }\end{array}$} & \multirow{2}{*}{$\begin{array}{c}\text { Participant } \\
\text { characteristics }\end{array}$} & \multirow{2}{*}{ Study design } & \multirow{2}{*}{$\begin{array}{l}\text { Length of } \\
\text { follow- } \\
\text { up }^{\mathrm{a}}\end{array}$} & \multirow{2}{*}{$\begin{array}{l}\text { Methods of } \\
\text { recruitment }\end{array}$} & \multicolumn{2}{|c|}{ Definitions and measurements } & \multicolumn{2}{|c|}{ Sample size } & \multirow{2}{*}{$\begin{array}{l}\text { Analysis method (incl. con- } \\
\text { trol of confounders) }\end{array}$} & \multirow{2}{*}{$\begin{array}{l}\text { Main findings regarding } \\
\text { the association between } \\
\text { adherence and abstinence }\end{array}$} \\
\hline & & & & & & adherence & success & Baseline & Follow-up & & \\
\hline (22) & $\begin{array}{l}\text { Countries: United } \\
\text { Kingdom \& USA } \\
\text { Year of study: not } \\
\text { reported } \\
\text { Population: partici- } \\
\text { pants of a smoking } \\
\text { cessation trial who } \\
\text { were abstinent during } \\
\text { the first two weeks }\end{array}$ & $\begin{array}{l}\text { mean age: } 43.3 \pm \\
12.1 \text { yrs } \\
55 \% \text { women } \\
94 \% \text { white } \\
\text { mean cig/d: } 21.0 \pm \\
10.0 \\
\text { mean FTND: } 4.1 \pm \\
2.4\end{array}$ & $\begin{array}{l}\text { secondary analysis of } \\
\text { an RCT of nicotine } \\
\text { lozenges ( } 2 \text { or } 4 \mathrm{mg} \text { ) } \\
\text { vs. placebo }\end{array}$ & 6 weeks & not reported & $\begin{array}{l}\text { adherence during the } \\
\text { first } 2 \text { weeks was } \\
\text { monitored daily (IVR } \\
\text { system) } \\
\text { definition of adherence: } \\
\text { 'high lozenge use' based } \\
\text { on a median split of all } \\
\text { participants; group } \\
\text { means: } 10.2 \pm 2.5 \text { vs. } \\
5.1 \pm 1.9 \text { lozenges per } \\
\text { day }\end{array}$ & $\begin{array}{l}\text { continuous } 28 \text {-day } \\
\text { abstinence at } \\
\text { week } 6 \text {, validated } \\
\text { by } \mathrm{CO}<10 \mathrm{ppm}\end{array}$ & $\begin{array}{l}1030 \text { of } \\
\text { which } 612 \\
\text { received } \\
\text { verum and } \\
418 \text { placebo }\end{array}$ & 1020 & $\begin{array}{l}\text { logistic regression for predictors of } 28- \\
\text { day abstinence at week } 6 \text {, adjusted for } \\
\text { gender and cig/d } \\
\text { control for relapse: exclusion of } \\
\text { participants who had smoked during } \\
\text { the first } 2 \text { study weeks }\end{array}$ & $\begin{array}{l}\text { OR of abstinence for high vs. low } \\
\text { lozenge use (participants in the } \\
\text { verum group only): } \\
\text { - unadjusted: } 1.60(1.13-2.27) \\
\text { - adjusted: } 1.25(1.05-1.50) \\
\text { OR of abstinence per additional } \\
\text { lozenge/day: } \\
\text { - unadjusted: } 1.10 \text { (1.04-1.16) } \\
\text { - adjusted: } 1.10(1.04-1.16) \\
\text { significant treatment-by-adherence } \\
\text { interaction }\end{array}$ \\
\hline (27) & $\begin{array}{l}\text { Country: United } \\
\text { Kingdom } \\
\text { Year of study: 2007- } \\
2009 \\
\text { Population: smokers } \\
\geq 10 / d\end{array}$ & $\begin{array}{l}\text { mean age } \sim 47 \text { yrs } \\
\sim 54 \% \text { women } \\
\sim 90 \% \text { white } \\
\sim 21 \text { cig/d } \\
\text { mean FTND } \sim 5.5\end{array}$ & $\begin{array}{l}\text { RCT of different ways } \\
\text { to tailor oral NRT in } \\
\text { addition to NRT } \\
\text { patches and counsel- } \\
\text { ling }\end{array}$ & 4 weeks & $\begin{array}{l}\text { Patients } \\
\text { attending one } \\
\text { of } 29 \text { primary } \\
\text { care practices } \\
\text { in Birmingham } \\
\text { \& Bristol were } \\
\text { directly } \\
\text { approached } \\
\end{array}$ & $\begin{array}{l}\text { Consumption }(\mathrm{mg} / \mathrm{d}) \\
\text { during the first week } \\
\text { was measured by self- } \\
\text { report and pill counts } \\
\text { and recorded in daily } \\
\text { diaries } \\
\text { Adherence }=\text { proportion } \\
\text { of prescribed dose } \\
\end{array}$ & $\begin{array}{l}\text { Self-reported } 4- \\
\text { week abstinence, } \\
\text { validated by } \\
\text { CO<10 ppm }\end{array}$ & 633 & 285 & $\begin{array}{l}\text { logistic regression for predictors of 4- } \\
\text { week abstinence, adjusted for trial } \\
\text { arm, genotype, cig/d, FTND, length of } \\
\text { previous quit attempts } \\
\text { control for relapse: exclusion of } \\
\text { participants who had smoked during } \\
\text { the first study week }\end{array}$ & $\begin{array}{l}\text { OR of abstinence per additional } \\
\mathrm{mg} / \mathrm{d} \text { consumed: } \\
\text {-unadjusted: } 1.03 \text { (0.99-1.07) } \\
\text { - adjusted: } 1.05(1.01-1.10)\end{array}$ \\
\hline (29) & $\begin{array}{l}\text { Country: USA } \\
\text { Year of study: 2004- } \\
2007 \\
\text { Population: patients } \\
\text { with a diagnosis of } \\
\text { alcohol abuse/ depen- } \\
\text { dence and smoking } \\
\geq 15 / d\end{array}$ & $\begin{array}{l}\text { mean age } \sim 45 \text { yrs } \\
\sim 29 \% \text { women } \\
\sim 90 \% \text { white } \\
\sim 25.5 \text { cig/d } \\
\text { mean FTND } \sim 6\end{array}$ & $\begin{array}{l}\text { RCT of nicotine gum vs. } \\
\text { placebo in addition to } \\
\text { a patch and behav- } \\
\text { ioural therapy }\end{array}$ & $\begin{array}{l}3,6 \text { and } 12 \\
\text { months }\end{array}$ & $\begin{array}{l}\text { radio \& news- } \\
\text { paper adver- } \\
\text { tisements } \\
\text { referrals from a } \\
\text { substance } \\
\text { abuse clinic }\end{array}$ & $\begin{array}{l}\text { no a priori definition of } \\
\text { adherence } \\
\text { measurement: } 7 \text {-day } \\
\text { retrospective report of } \\
\text { the frequency of gum } \\
\text { use, assessed } 2 \text { wks } \\
\text { after target quit date }\end{array}$ & $\begin{array}{l}\text { self-reported } \\
\text { continuous } \\
\text { abstinence at all } \\
\text { time points, } \\
\text { validated by co } \\
<10 \mathrm{ppm}\end{array}$ & $\begin{array}{l}96 \text { of which } \\
45 \text { received } \\
\text { verum }\end{array}$ & $\begin{array}{l}\text { follow-up data } \\
\text { based on the } \\
\text { verum group (n } \\
=45 \text { ): } \\
3 \text { months: } 37 \\
6 \text { months: } 32 \\
12 \text { months: } 30\end{array}$ & $\begin{array}{l}\text { logistic regression for continuous } \\
\text { abstinence at different time-points, } \\
\text { adjusted for education level, depres- } \\
\text { sion score, FTND and study site; } \\
\text { control for relapse: Assessment of } \\
\text { adherence at } 2 \text { weeks included only } \\
\text { those who were still abstinent }(n=37)\end{array}$ & $\begin{array}{l}\text { adjusted ORs for continuous } \\
\text { abstinence at... } \\
-3 \text { months: } 1.04(1.01-1.06) \\
-6 \text { months: } 1.04(1.01-1.08) \\
-12 \text { months: } 1.03 \text { (0.97-1.10) }\end{array}$ \\
\hline (28) & $\begin{array}{l}\text { Country: Germany } \\
\text { Year of study: 2003- } \\
2006 \\
\text { Population: smokers } \\
\text { (general population } \\
\text { and hospital staff) } \\
\end{array}$ & $\begin{array}{l}\text { median age: } 45 \text { yrs } \\
58.8 \% \text { women } \\
\text { median cid/g: } 20 \\
\text { median FTND: } 5\end{array}$ & $\begin{array}{l}\text { prospective observa- } \\
\text { tional study of a } \\
\text { hospital-based } \\
\text { smoking cessation } \\
\text { clinic; participants self- } \\
\text { selected to use NRT }\end{array}$ & 6 months & $\begin{array}{l}\text { local newspa- } \\
\text { per articles, } \\
\text { posters and } \\
\text { flyers }\end{array}$ & $\begin{array}{l}\text { no a priori definition of } \\
\text { adherence }\end{array}$ & $\begin{array}{l}\text { self-reported } \\
\text { continuous 6- } \\
\text { month abstinence; } \\
\text { no biochemical } \\
\text { validation }\end{array}$ & $\begin{array}{l}369 \text { of which } \\
182 \text { self- } \\
\text { selected to } \\
\text { use NRT }\end{array}$ & $\begin{array}{l}127 \text { partici- } \\
\text { pants used NRT } \\
\text { and did not } \\
\text { relapse before } \\
\text { stopping NRT }\end{array}$ & $\begin{array}{l}\chi^{2} \text { test } \\
\text { control for relapse: participants who } \\
\text { relapsed before stopping NRT were } \\
\text { excluded }\end{array}$ & $\begin{array}{l}\text { continuous } 6 \text {-month abstinence } \\
\text { rates for NRT use }>35 \text { days vs. }<35 \\
\text { days: } 61.0 \% \text { vs. } 42.6 \% ; p=0.039\end{array}$ \\
\hline (26) & $\begin{array}{l}\text { Country: Switzerland } \\
\text { Year of study: 1996- } \\
1997 \\
\text { Population: smokers } \\
\geq 15 / d\end{array}$ & $\begin{array}{l}\text { median age: } 40 \text { yrs } \\
46.7 \% \text { women } \\
\text { median cig/d: } 25 \\
\text { median FTND: } 5\end{array}$ & $\begin{array}{l}\text { prospective observa- } \\
\text { tional study of } \\
\text { prolonged use (up to } \\
18 \text { months) of nicotine } \\
\text { nasal spray }\end{array}$ & 24 months & $\begin{array}{l}\text { referrals to the } \\
\text { smoking } \\
\text { cessation unit } \\
\text { and advertise- } \\
\text { ments in a } \\
\text { hospital } \\
\end{array}$ & $\begin{array}{l}\text { no a priori definition of } \\
\text { adherence } \\
\text { MDllog: adherence was } \\
\text { only assessed for the } \\
\text { first month }\end{array}$ & $\begin{array}{l}\text { self-reported } \\
\text { continuous } \\
\text { abstinence from } \\
\text { mo } 1 \text { to mo } 24, \\
\text { valiated by } \\
\text { co } 10 \mathrm{ppm} \\
\end{array}$ & 92 & $\begin{array}{l}82 \text { of which } 48 \\
\text { were 'totally } \\
\text { abstinent' after } \\
1 \text { month }\end{array}$ & $\begin{array}{l}\text { multiple logistic regression for } \\
\text { predictors of abstinence } \\
\text { control for relapse: pts. who smoked } \\
\text { at } 1 \text { mo were excluded }\end{array}$ & $\begin{array}{l}\text { Median daily consumption of nasal } \\
\text { spray was not predictive of absti- } \\
\text { nence (no ORs provided). }\end{array}$ \\
\hline
\end{tabular}

Table 1: Characteristics of included studies. yrs, years; cig/d, cigarettes per day; FTND, Fagerström Test of Nicotine Dependence; RCT, randomised controlled trial; NRT, nicotine replacement therapy; MDILog, metered-dose inhaler chronolog; IVR, interactive voice response; CO, carbon monoxide; ppm, parts per million; PP, point prevalence; pts, patients; OR, odds ratio

${ }^{a}$ Unless otherwise stated, length of follow-up refers to the time point used to establish the association between adherence and abstinence 


\section{Figures}

Figure 1: Flowchart of the study selection and exclusion process

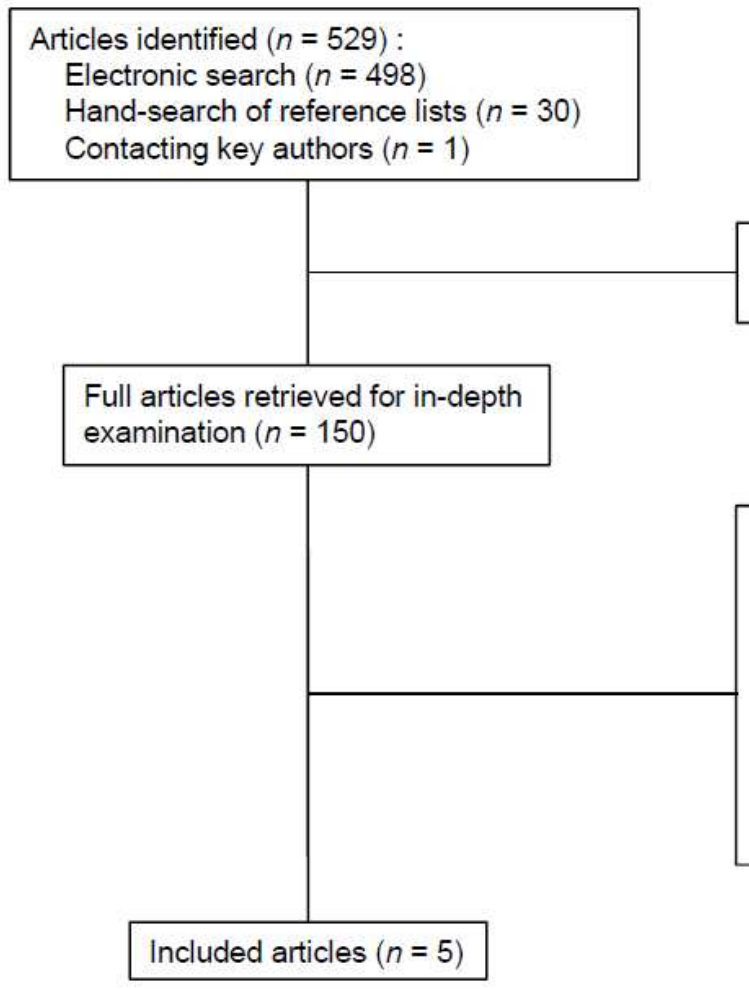

Excluded on the basis of title and abstract $(n=379)$
Excluded $(n=145)$ :
Unrelated to smoking cessation $(n=7)$
Unrelated to pharmacotherapy $(n=11)$
Unrelated to adherence $(n=17)$
Studies only including adolescents $(n=1)$
No assessment of the association between adherence and abstinence $(n=72)$
No control for relapse as a potential cause of non-adherence $(n=37)$




\section{Supporting information}

Additional Supporting Information may be found in the online version of this article:

Table S1: Characteristics of studies which were excluded due to a lack of control for confounding by non-adherence due to relapse. yrs, years; cig/d, cigarettes per day; FTND, Fagerström Test of Nicotine Dependence; RCT, randomised controlled trial; NRT, nicotine replacement therapy; MDILog, metered-dose inhaler chronolog; IVR, interactive voice response; CO, carbon monoxide; ppm, parts per million; PP, point prevalence; pts, patients; OR, odds ratio

Appendix S1: Systematic review protocol 


\begin{tabular}{|c|c|c|c|c|c|c|c|c|c|c|c|}
\hline \multirow{2}{*}{ ID } & \multirow{2}{*}{$\begin{array}{l}\text { Country, Year } \\
\text { and Study } \\
\text { Population }\end{array}$} & \multirow{2}{*}{$\begin{array}{l}\text { Participant } \\
\text { characteristics }\end{array}$} & \multirow{2}{*}{$\begin{array}{l}\text { Design \& data } \\
\text { collection }\end{array}$} & \multirow{2}{*}{$\begin{array}{l}\text { Recall period } \\
\text { / length of } \\
\text { follow-up }\end{array}$} & \multirow{2}{*}{$\begin{array}{l}\text { Methods of } \\
\text { recruitment }\end{array}$} & \multicolumn{2}{|c|}{ Definitions } & \multicolumn{2}{|c|}{ Sample size } & \multirow{2}{*}{$\begin{array}{l}\text { Analysis method } \\
\text { (incl. control of } \\
\text { confounders) }\end{array}$} & \multirow{2}{*}{$\begin{array}{l}\text { Main findings regarding } \\
\text { the association between } \\
\text { adherence and abstinence }\end{array}$} \\
\hline & & & & & & adherence & success & Baseline & Follow-up & & \\
\hline [1] & $\begin{array}{l}\text { USA } \\
\text { before 1999 } \\
\text { smokers }(\geq 20 / d) \\
\text { participating in a } \\
\text { university-based } \\
\text { cessation clinic }\end{array}$ & $\begin{array}{l}38.7 \pm 10.2 \mathrm{yrs} \\
53.5 \% \text { women } \\
62.4 \% \text { Caucas. } \\
26.4 \pm 10.3 \mathrm{cig} / \mathrm{d} \\
\text { FTND } 6.7 \pm 1.5\end{array}$ & $\begin{array}{l}\text { 8-week patch Tx plus } \\
\text { three levels of support } \\
\text { (random.) } \\
\text { questionnaires on } \\
\text { withdrawal, motivation } \\
\text { (URICA), self-efficacy }\end{array}$ & $\begin{array}{l}\text { week } 9 \text { and week } \\
26 \\
\text { timeline follow- } \\
\text { back (TLFB) } \\
\text { method }\end{array}$ & $\begin{array}{l}\text { University } \\
\text { campus } \\
\text { notices, } \\
\text { newspaper ads, } \\
\text { 'word of mouth }\end{array}$ & $\begin{array}{l}\text { continuously wearing } \\
\text { the assigned } \\
\text { patch at the recom- } \\
\text { mended patch dose } \\
\text { in the } \\
\text { instructed manner } \\
\text { for the entire } 24-h \\
\text { time period } \\
\text { dichotomised } \\
\text { measure of adher- } \\
\text { ence = patch use for } \\
\text { more than the } \\
\text { median number of } \\
\text { days }\end{array}$ & $\begin{array}{l}\text { self-report (TLFB) of } \\
\text { abstinence of } \\
\text { unspecified duration, } \\
\text { validated by } \mathrm{CO}<10 \\
\mathrm{ppm}\end{array}$ & $\begin{array}{l}101(34 / 34 / 33 \text { in } \\
\text { the } 3 \text { groups) }\end{array}$ & $\begin{array}{l}\text { "11 to } 12 \text { treat- } \\
\text { ment drop-outs", } \\
\text { a majority of } \\
\text { whom were } \\
\text { smoking at the 9- } \\
\text { and 26-week } \\
\text { follow-up! }\end{array}$ & $\begin{array}{l}\text { correlation analysis and } \\
\text { logistic regression for } \\
\text { predictors of abstinence } \\
\text { no specific control for } \\
\text { non-adherence due to } \\
\text { relapse }\end{array}$ & $\begin{array}{l}\text { Week 9: correlation patch use / } \\
\text { smoking: r=-0.50; p<0.0005; } \\
\text { dichotomised aherence / absti- } \\
\text { nence: unadjusted OR 23.44 (6.51- } \\
\text { 84.39); adjusted OR } 3.22 \text { (0.94- } \\
\text { 11.07) } \\
\text { Week 26: correlation patch use / } \\
\text { smoking: } r=-0.34 ; p=0.002 \text {; dichot- } \\
\text { omised adherence / abstinence: } \\
\text { unadjusted OR 4.25 (1.28-14.12); } \\
\text { adjusted OR } 1.43(0.33-6.18)\end{array}$ \\
\hline [2] & $\begin{array}{l}\text { Australia, Canada, } \\
\text { United Kingdom, USA } \\
2006-2008 \\
\text { smokers or recent } \\
\text { quitters who had used } \\
\text { medication in the } \\
\text { previous year }\end{array}$ & $\begin{array}{l}45.5 \pm 13.0 \text { yrs } \\
60.5 \% \text { women } \\
17.5 \pm 9.2 \text { cig/d } \\
\text { NRT use: } 80.5 \% \text { (of } \\
\text { these: OTC } 68.3 \% \text { ) } \\
\text { BUP/NAR use: } \\
19.5 \%\end{array}$ & $\begin{array}{l}\text { ITC Four-Country } \\
\text { Survey (CAN, UK, USA, } \\
\text { and AUS), } \\
\text { computer-assisted } \\
\text { telephone interviews } \\
\text { Waves } 5 \& \text { \& } 6(10 / 06- \\
\text { 2/08), including only } \\
\text { follow-ups that had } \\
\text { been recruited at least } \\
\text { one wave before }\end{array}$ & $\begin{array}{l}12 \text { months } \\
\text { preceding the } \\
\text { interview (retro- } \\
\text { spective) }\end{array}$ & $\begin{array}{l}\text { recruitment of } \\
\text { smokers using } \\
\text { random-digit } \\
\text { dialling }\end{array}$ & $\begin{array}{l}\geq 8 \text { weeks of treat- } \\
\text { ment with NRT, BUP } \\
\text { or VAR (sub-groups: } \\
<1 \mathrm{wk}, 1-2 \text { wks, } 2-4 \\
\text { wks, } 4-8 \text { wks, } \geq 8 \text { wks) } \\
\text { NRT: adequate dose: } \\
\geq 10 \text { pcs/d }\end{array}$ & $\begin{array}{l}\text { self-reported 6- } \\
\text { month continuous } \\
\text { abstinence; no } \\
\text { biochemical valida- } \\
\text { tion }\end{array}$ & $\begin{array}{l}1219(920 \\
\text { relapsers, } 299 \\
\text { successful } \\
\text { quitters) }\end{array}$ & $\begin{array}{l}\text { use duration data } \\
\text { available for } 1118 \\
\text { subjects; } \\
\text { smoking outcome } \\
\text { data available for } \\
548 \text { subjects; of } \\
\text { these, 22.6\% } \\
\text { achieved 6\%-mo } \\
\text { cont. abstin., but } \\
201 \text { were exclud- } \\
\text { ed (discontin. due } \\
\text { to relapse) } \\
\end{array}$ & $\begin{array}{l}\text { multiple logistic } \\
\text { regression for predic- } \\
\text { tors of abstinence; all } \\
\text { subjects who recalled } \\
\text { having discontinued due } \\
\text { to relapse were } \\
\text { excluded (risk of recall } \\
\text { bias) }\end{array}$ & $\begin{array}{l}\text { adjusted ORs for continuous } \\
\text { abstinence at } 6 \text { mo (data for } n= \\
\text { 347): } \\
\text { - - 'no-adherences: } 0.16(\text { (o.08-0.0.31) } \\
- \text { 'not needed' 'ss other reasons: } \\
3.26(1.75-6.07)\end{array}$ \\
\hline [3] & $\begin{array}{l}\text { USA } \\
2006-2007 \\
\text { smokers } \geq 10 / d\end{array}$ & $\begin{array}{l}47.3 \pm 10.9 \mathrm{yrs} \\
66.8 \% \text { women } \\
89.6 \% \text { white } \\
19.7 \pm 8.2 \mathrm{cig} / \mathrm{d} \\
\text { FTND } 5.0 \pm 2.1\end{array}$ & $\begin{array}{l}\text { secondary analysis of } \\
\text { the COMPASS Trial } \\
\text { (VAR): } \\
\text { smokers who set a quit } \\
\text { date were mailed one } \\
\text { 28-day supply of VAR; } \\
\text { three levels of support } \\
\text { (random.) }\end{array}$ & $\begin{array}{l}\text { telephone } \\
\text { interviews at } \\
\text { baseline, } 21 \text { days, } \\
12 \text { weeks, and } 6 \\
\text { months post } \\
\text { target quit date }\end{array}$ & $\begin{array}{l}\text { brochures } \\
\text { placed in health } \\
\text { plan-owned } \\
\text { clinics, physi- } \\
\text { cian referrals, } \\
\text { Quit For Life } \\
\text { Program }\end{array}$ & $\begin{array}{l}4 \text { self-report indices: } \\
\text { a) days taken } \\
\text { (dichotomised: } \geq 80 \% \\
\text { of prescribed } \\
\text { amount) } \\
\text { b) taken for } 7 \text { days } \\
\text { before TQD } \\
\text { c) taken for } 7 \text { days } \\
\text { atter TQD } \\
\text { d) Medication } \\
\text { Adherence Ques- } \\
\text { tionnaire (intestional } \\
\text { vs. unintentional } \\
\text { non-adherence) }\end{array}$ & $\begin{array}{l}\text { self-reported 7-day } \\
\text { PP; no biochemical } \\
\text { validation }\end{array}$ & 1161 & $\begin{array}{l}893 \text { to } 1161 \\
\text { (depending on the } \\
\text { adherence index } \\
\text { examined); } \\
\text { strange: the } \\
\text { mother paper } \\
\text { reports a } 6 \text {-month } \\
\text { sample size of } 892\end{array}$ & $\begin{array}{l}\text { logistic regression for } \\
\text { predictors of 6-month } \\
\text { continuous abstinence } \\
\text { (using the } 4 \text { adherences } \\
\text { indices) } \\
\chi^{2} \text { Test for assoc. btw. } \\
\text { dichotomised adher- } \\
\text { ence and 7-day PP at } 6 \\
\text { months } \\
\text { no specific control for } \\
\text { non-adherence due to } \\
\text { relapse }\end{array}$ & $\begin{array}{l}\text { unadjusted ORs for } 7 \text {-day PP at } 6 \\
\text { months per } 1 \mathrm{SD} \text { increase in the } \\
\text { independent variable: } \\
\text { a) } 1.81(1.56-2.09) \\
\text { b) } 1.15(1.00-1.33) \\
\text { c) } 1.24(1.07-1.44) \\
\text { d) less intentional non-adherence: } \\
1.23(1.07-1.41) \\
\text { prevalence of } 7 \text {-day PP at } 6 \text { months: } \\
52.2 \% \text { (adherent) vs. } 25.4 \% \text { (non- } \\
\text { adherent); p<0.0001 }\end{array}$ \\
\hline [4] & $\begin{array}{l}\text { USA } \\
2003-2005 \\
\text { smokers } \geq 10 / d\end{array}$ & $\begin{array}{l}42.7 \pm 11.5 \mathrm{yrs} \\
51.8 \% \text { women } \\
81.9 \% \text { white } \\
22.6 \pm 9.3 \mathrm{cid} / \mathrm{d} \\
\text { FTND } 5.4 \pm 2.1\end{array}$ & $\begin{array}{l}\text { secondary analysis of } \\
\text { an RCT of message } \\
\text { framing for smoking } \\
\text { cessation in addition to } \\
\text { BUP }\end{array}$ & $\begin{array}{l}\text { timeline follow- } \\
\text { back (TLFB) } \\
\text { method } \\
\text { maximum follow- } \\
\text { up } 26 \text { weeks }\end{array}$ & $\begin{array}{l}\text { newspaper and } \\
\text { radio ads, } \\
\text { press releases, } \\
\text { mailings to } \\
\text { physicians, } \\
\text { Internet }\end{array}$ & $\begin{array}{l}\text { Percentage adher- } \\
\text { ence = number of } \\
\text { cap openings } / 95 * \\
100 \\
\text { "Treatment comple- } \\
\text { tion" is mentioned } \\
\text { but not defined }\end{array}$ & $\begin{array}{l}\text { self-reported } 7-d \mathrm{PP} \\
\text { at } 6,12 \text { and } 26 \\
\text { weeks, validated by } \\
\mathrm{CO} \leq 10 \mathrm{ppm}\end{array}$ & 249 & 249 & $\begin{array}{l}\text { linear and logistic } \\
\text { regression, adjusted for } \\
\text { message framing (i.e., } \\
\text { rando group) } \\
\text { no specific control for } \\
\text { non-adherence due to } \\
\text { relapse }\end{array}$ & $\begin{array}{l}\text { ORs of quitting at various time- } \\
\text { points, by mean percentage of cap } \\
\text { openings: } \\
-6 \text {-wk cont. abst.: } 0.96(0.95-0.98) \\
\text { - 7-d PP at } 6 \text { wks: } 0.96(0.95-0.97) \\
\text {-7 -d PP at } 12 \text { wks: } 0.97(0.96-0.99) \\
\text {-7-d PP at } 26 \text { wks: } 0.97(0.95-0.99)\end{array}$ \\
\hline [5] & $\begin{array}{l}\text { USA } \\
2003-2005 \\
\text { smokers } \geq 10 / d\end{array}$ & $\begin{array}{l}42.9 \pm 11.6 \mathrm{yrs} \\
44.0 \% \text { women } \\
81.6 \% \text { white } \\
21.6 \pm 9.1 \mathrm{cid} / \mathrm{d} \\
\text { FTND } 5.3 \pm 2.1 \\
\end{array}$ & $\begin{array}{l}\text { secondary analysis of } 2 \\
\text { RCTs of VAR/BUP/PLC } \\
\text { for smoking cessation }\end{array}$ & 12 weeks & $\begin{array}{l}\text { media advertis- } \\
\text { ing }\end{array}$ & $\begin{array}{l}\text { "completer": } \\
\text { subjects who took } \geq 1 \\
\text { dose of medication } \\
\text { for } \geq 80 \% \text { of the } \\
\text { treatment days }\end{array}$ & $\begin{array}{l}\text { self-reported } \\
\text { abstinence wks 9-12, } \\
\text { validated by } \mathrm{CO} \leq 10 \\
\text { ppm }\end{array}$ & $\begin{array}{l}2045 \text { (692 VAR, } \\
669 \text { BUP, } 684 \text { PLC) }\end{array}$ & 2045 & $\begin{array}{l}\text { logistic regression for } \\
\text { predictors of abstinence } \\
\text { no specific control for } \\
\text { non-adherence due to } \\
\text { relapse }\end{array}$ & $\begin{array}{l}\text { positive correlation between } \\
\text { adherence to treatment and } \\
\text { tobacco abstinence with no } \\
\text { significant treatment-by-adherence } \\
\text { interaction (data presented in a }\end{array}$ \\
\hline
\end{tabular}




\begin{tabular}{|c|c|c|c|c|c|c|c|c|c|c|c|}
\hline & & & & & & $\begin{array}{l}\text { during the 12-week } \\
\text { treatment period }\end{array}$ & & & & & figure; no ORs reported) \\
\hline [6] & $\begin{array}{l}\text { China } \\
\text { 2000-2002 } \\
\text { smokers attending a } \\
\text { Smoking Cessation } \\
\text { Health Centre }\end{array}$ & $\begin{array}{l}85 \% \text { aged } 20-59 \\
20 \% \text { women } \\
72 \% \text { smoked }>10 / \text { d } \\
43 \% \text { with FTND } 25\end{array}$ & $\begin{array}{l}\text { Prospective observa- } \\
\text { tional study 8/00-1/02; } \\
\text { the cessation service } \\
\text { (including a 1-wk } \\
\text { supply of NRT) was } \\
\text { free }\end{array}$ & $\begin{array}{l}\text { follow-up } \\
\text { interviews } \\
\text { including self- } \\
\text { administered } \\
\text { questionnaires at } \\
1,3 \text { and 12 } \\
\text { months } \\
\text { NRT use was } \\
\text { asked about at 3 } \\
\text { months retrospec- } \\
\text { tively } \\
\end{array}$ & $\begin{array}{l}\text { subjects } \\
\text { reporting to the } \\
\text { centre via } \\
\text { phone or in } \\
\text { person were } \\
\text { invited to } \\
\text { participate }\end{array}$ & $\begin{array}{l}\text { NRT use for } \geq 4 \mathrm{wks} \\
\text { during the first } 3 \\
\text { months (self-report } \\
\text { at } 3 \text { months) }\end{array}$ & $\begin{array}{l}\text { self-reported 7-d PP } \\
\text { at } 12 \text { months; } n \\
\text { biochemical valida- } \\
\text { tion }\end{array}$ & $\begin{array}{l}1186 \text { of which } \\
89 \% \text { received a } \\
\text { prescription for } \\
\text { NRT } \rightarrow 1051\end{array}$ & $\begin{array}{l}3 \text { months: } 889 \\
12 \text { months: } 698\end{array}$ & $\begin{array}{l}\text { logistic regression for } \\
\text { predictors of abstinence } \\
\text { no specific control for } \\
\text { non-adherence due to } \\
\text { relapse }\end{array}$ & 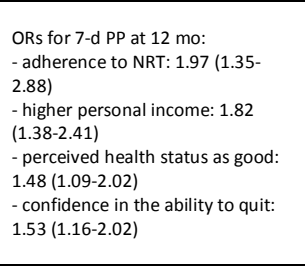 \\
\hline [7] & $\begin{array}{l}\text { USA } \\
1992 \\
\text { elderly smokers (65- } \\
74 \text { yrs) }\end{array}$ & $\begin{array}{l}69.3 \pm 2.7 \text { yrs } \\
75.2 \% \text { women } \\
92.2 \% \text { white } \\
\text { no data on } \\
\text { baseline smoking } \\
\text { due to an error }\end{array}$ & $\begin{array}{l}\text { telephone survey; } \\
\text { inclusion during the } \\
\text { first quarter of } 1992\end{array}$ & $\begin{array}{l}\text { telephone } \\
\text { interview 6 } \\
\text { months after NRT } \\
\text { use (retrospec- } \\
\text { tive) }\end{array}$ & $\begin{array}{l}\text { Patients filing } \\
\text { new claims for } \\
\text { nicotine } \\
\text { patches } \\
\text { through the } \\
\text { PACE program } \\
\text { were invited }\end{array}$ & $\begin{array}{l}\text { no a priori definition } \\
\text { of adherence }\end{array}$ & $\begin{array}{l}\text { self-reported PP at } 6 \\
\text { months; no biochem- } \\
\text { ical validation }\end{array}$ & $\begin{array}{l}1070 \text { of which } 940 \\
\text { turned out to be } \\
\text { eligible }\end{array}$ & $\begin{array}{l}871 \text { pts. complet- } \\
\text { ed the interview }\end{array}$ & $\begin{array}{l}\text { T test to compare } \\
\text { quitters and non- } \\
\text { quitters regarding the } \\
\text { duration of NRT patch } \\
\text { use } \\
\text { no specific control for } \\
\text { non-adherence due to } \\
\text { relapse }\end{array}$ & $\begin{array}{l}\text { duration of patch use in quitters vs. } \\
\text { non-quitters: } 48.4 \pm 31.9 \text { days vs. } \\
32.0 \pm 33.1 \text { days }(p<0.001)\end{array}$ \\
\hline [8] & $\begin{array}{l}\text { United Kingdom } \\
2007-2008 \\
\text { adults who had } \\
\text { received prescriptions } \\
\text { for varenicline }\end{array}$ & $\begin{array}{l}\text { mean age } 46.5 \text { yrs } \\
60.6 \% \text { women }\end{array}$ & $\begin{array}{l}\text { THIN database: mailed } \\
\text { questionnaire survey }\end{array}$ & $\begin{array}{l}\text { approximately } 6 \\
\text { months, retro- } \\
\text { spective }\end{array}$ & $\begin{array}{l}\text { data extraction } \\
\text { (random } \\
\text { sample) from } \\
\text { an existing } \\
\text { database }\end{array}$ & $\begin{array}{l}\text { no a priori definition } \\
\text { of adherence }\end{array}$ & $\begin{array}{l}\text { self-reported 7-d PP } \\
\text { at approximately } 6 \\
\text { months; no biochem- } \\
\text { ical validation }\end{array}$ & 915 & 193 & $\begin{array}{l}\text { univariate logistic } \\
\text { regression for predic- } \\
\text { tors of 6-month } \\
\text { abstinence; no specific } \\
\text { control for non- } \\
\text { adherence due to } \\
\text { relapse } \\
\end{array}$ & $\begin{array}{l}\text { ORs of abstinence for various } \\
\text { treatment durations (ref: }<2 \\
\text { weeks): } \\
-2-2 \text { wks: } 2.0(0.6-6-1) \\
-5-8 \text { wks: } 5.4(1.9-15.9) \\
-9-12 \text { wks: } 11.0(3.9-31.1) \\
->12 \text { wks: } 7.6(2.0-28.8) \\
\end{array}$ \\
\hline [9] & $\begin{array}{l}\text { Country: USA } \\
\text { Year of study: not } \\
\text { reported } \\
\text { Population: smokers }\end{array}$ & $\begin{array}{l}\text { mean age: } 42.8 \pm \\
11.5 \text { yrs } \\
52.6 \% \text { women } \\
87.3 \% \text { white } \\
\text { mean cig/d: } 25.2 \pm \\
11.3\end{array}$ & $\begin{array}{l}\text { secondary analysis of } \\
\text { an RCT of nicotine } \\
\text { patches vs. placebo } \\
\text { under simulated over- } \\
\text { the-counter conditions }\end{array}$ & 6 weeks & not reported & $\begin{array}{l}\text { adherence during } \\
\text { wks } 1-3 \text { was cap- } \\
\text { tured in a patient } \\
\text { diary } \\
\text { definition of adher- } \\
\text { ence: } \\
\text { a) patch use for } \geq 20 \\
\text { of } 21 \text { treatment days } \\
\text { (post-hoc definition) } \\
\text { b) linear measure } \\
\end{array}$ & $\begin{array}{l}\text { self-reported } 7-d \text { PP } \\
\text { at } 6 \text { weeks, validated } \\
\text { by } C O<10 \text { ppm }\end{array}$ & 567 & 371 & $\begin{array}{l}\text { logistic regression for } \\
\text { predictors of abstinence } \\
\text { at } 6 \text { weeks } \\
\text { control for relapse: The } \\
\text { model controlled for } \\
\text { smoking status during } \\
\text { weeks 1-3 }\end{array}$ & $\begin{array}{l}\text { OR of abstinence in adherent vs. } \\
\text { non-adherent subjects (active group } \\
\text { only): } 3.25(1.30-8.09) ; 53.2 \% \text { vs. } \\
21.5 \% \\
\text { significant treatment-by-adherence } \\
\text { interaction } \\
\text { similar results when a linear } \\
\text { measure of adherence was used }\end{array}$ \\
\hline [10] & $\begin{array}{l}\text { USA } \\
1992 \\
\text { patients who received } \\
\text { a nicotine patch } \\
\text { prescription }\end{array}$ & $\begin{array}{l}\text { mean age } \sim 40 \text { yrs } \\
57 \% \text { women }\end{array}$ & $\begin{array}{l}\text { Telephone interviews } \\
\text { between } 9 / 92 \text { and } \\
11 / 92\end{array}$ & $\begin{array}{l}\text { between } 3 \text { and } 10 \\
\text { months (not } \\
\text { specified), } \\
\text { retrospective }\end{array}$ & $\begin{array}{l}\text { data extraction } \\
\text { from an } \\
\text { existing } \\
\text { database }\end{array}$ & $\begin{array}{l}\text { no a priori definition } \\
\text { of adherence }\end{array}$ & $\begin{array}{l}\text { self-reported PP at } \\
\text { the time of the } \\
\text { interview; no } \\
\text { biochemical valida- } \\
\text { tion }\end{array}$ & eligible: 404 & $\begin{array}{l}\text { completed the } \\
\text { interview: } 284 ; \\
\text { subsample used: } \\
260\end{array}$ & $\begin{array}{l}\text { within-group compari- } \\
\text { son (non-smokers) using } \\
\text { T and } \chi^{2} \text { tests; } \\
\text { no specific control for } \\
\text { non-adherence due to } \\
\text { relapse }\end{array}$ & $\begin{array}{l}\text { Nonsmokers were more likely to } \\
\text { have used the patch longer than } \\
\text { shorter (5-level variable) and to } \\
\text { report having used the patch every } \\
\text { day as compared to less frequently } \\
\text { (3-level variable) }\end{array}$ \\
\hline [11] & $\begin{array}{l}\text { Sweden } \\
\text { before } 1984 \\
\text { no further infor- } \\
\text { mation }\end{array}$ & $\begin{array}{l}\text { mean age: } 40.7 \text { yrs } \\
56 \% \text { women } \\
\text { mean cig/d: } 19.0 \\
\text { mean FTND: } 6.3\end{array}$ & $\begin{array}{l}\text { RCT of long vs. short } \\
\text { support and gum vs. } \\
\text { no gum (2x2 design) }\end{array}$ & $\begin{array}{l}\text { mailed question- } \\
\text { naire or phone call } \\
\text { at } 6 \& 12 \text { months }\end{array}$ & $\begin{array}{l}\text { enrolment } \\
\text { through } \\
\text { participating } \\
\text { physicians }\end{array}$ & $\begin{array}{l}\text { "arbitrary criterion": } \\
\text { chewing } \geq 10 \text { pieces } \\
\text { per day }\end{array}$ & $\begin{array}{l}\text { self-reported PP at } 6 \\
\text { and } 12 \text { months, } \\
\text { validated by CO } \leq 4 \\
\text { ppm at } 6 \text { mo in a } \\
\text { subsample }(n=26)\end{array}$ & 151 & 145 & $\begin{array}{l}\text { descriptive analysis } \\
\text { no specific control for } \\
\text { non-adherence due to } \\
\text { relapse }\end{array}$ & $\begin{array}{l}\text { PP at } 12 \text { mo for adherent vs. non- } \\
\text { adherent pts: } 30 \% \text { vs. } 22 \%\end{array}$ \\
\hline [12] & $\begin{array}{l}\text { USA } \\
\text { before } 1989 \\
\text { smokers } \geq 1 \text { pack/day }\end{array}$ & $\begin{array}{l}\text { mean age } \sim 42 \text { yrs } \\
\sim 53 \% \text { women } \\
\sim 27 \text { cig/d } \\
\text { mean FTND } \sim 6.5\end{array}$ & $\begin{array}{l}\text { RCT of behavioural Tx } \\
\text { vs. 'education' and } \\
\text { fixed vs. ad lib gum } \\
(2 \times 2 \text { design); the } \\
\text { treatment phase lasted } \\
11 \text { weeks }\end{array}$ & 6 months & newspaper ads & $\begin{array}{l}\text { compliance index: } \\
\text { pieces chewed / } \\
\text { pieces scheduled } \\
\text { An index of }>0.6 \text { was } \\
\text { considered 'suffi- } \\
\text { cient' }\end{array}$ & $\begin{array}{l}\text { self-reported } 48 \text {-h } \\
\mathrm{PP} \text {, validated by } \\
\mathrm{CO}<8 \mathrm{ppm} \text { at } 10 \text { wks } \\
\text { and by saliva cotinine } \\
<10 \mathrm{ng} / \mathrm{ml} \text { at } 6 \\
\mathrm{months}\end{array}$ & 107 & $\begin{array}{l}89 \text { of which } 82 \\
\text { provided data at } 6 \\
\text { months }\end{array}$ & $\begin{array}{l}\text { descriptive analysis } \\
\text { no specific control for } \\
\text { non-adherence due to } \\
\text { relapse }\end{array}$ & $\begin{array}{l}\text { "Average gum use did not correlate } \\
\text { significantly with any outcome } \\
\text { variable" (no data provided, except } \\
\text { for a subgroup analysis for } n=10 \\
\text { with no p values in Table 2) }\end{array}$ \\
\hline [13] & $\begin{array}{l}\text { Italy } \\
2007 \text {-2008 } \\
\text { smokers motivated to } \\
\text { quit }\end{array}$ & $\begin{array}{l}51.1 \pm 10.7 \mathrm{yrs} \\
56.3 \% \text { women } \\
22.8 \pm 8.8 \mathrm{cig} / \mathrm{d} \\
\text { mean FTD } \sim 5.5\end{array}$ & $\begin{array}{l}\text { non-randomised trial } \\
\text { of VAR vs. PLC (self- } \\
\text { selection) in addition } \\
\text { to a 6-wk group } \\
\text { cessation course }\end{array}$ & $\begin{array}{l}12,26 \text { and } 52 \\
\text { weeks }\end{array}$ & $\begin{array}{l}\text { outpatient } \\
\text { clinic }\end{array}$ & $\begin{array}{l}\text { taking VAR for } 12 \\
\text { weeks }\end{array}$ & $\begin{array}{l}\text { self-reported PP, } \\
\text { validated by CO<10 } \\
\text { ppm in } 22 \text { subjects at } \\
12 \text { months }\end{array}$ & 112 & $\begin{array}{l}110 \text { of which } 48 \\
\text { self-selected to } \\
\text { take VRR and } \\
\text { were included in } \\
\text { the analysis }\end{array}$ & $\begin{array}{l}\text { propensity score } \\
\text { matching (to account } \\
\text { for self-selection) } \\
\chi^{2} \text { test } \\
\text { no specific control for } \\
\text { non-adherence due to }\end{array}$ & $\begin{array}{l}\text { PP at } 12 \text { months: } 62.5 \% \text { (adherent) } \\
\text { vs. } 53.1 \% \text { (non-adherent); } p=0.381\end{array}$ \\
\hline
\end{tabular}




\begin{tabular}{|c|c|c|c|c|c|c|c|c|c|c|c|}
\hline & & & & & & & & & & $\begin{array}{l}\text { relapse } \\
\end{array}$ & \\
\hline [14] & $\begin{array}{l}\text { USA } \\
\text { 2003-2006 } \\
\text { heavy drinking } \\
\text { smokers } \geq 10 / d\end{array}$ & $\begin{array}{l}41.5 \pm 12.0 \mathrm{yrs} \\
45 \% \text { women } \\
90.7 \% \text { white } \\
21.3 \pm 9.4 \mathrm{cig} / \mathrm{d} \\
\text { FTND } 5.0 \pm 2.2\end{array}$ & $\begin{array}{l}\text { RCT of adding a brief } \\
\text { alcohol intervention to } \\
\text { a 4-week individual } \\
\text { smoking cessation } \\
\text { treatment; all pts. } \\
\text { received NRT patches }\end{array}$ & $\begin{array}{l}\text { maximum follow- } \\
\text { up } 26 \text { weeks }\end{array}$ & $\begin{array}{l}\text { community } \\
\text { bulletin boards, } \\
\text { newspaper \& } \\
\text { radio ads }\end{array}$ & $\begin{array}{l}\text { Percent days of using } \\
\text { patch during was } \\
\text { used as the index of } \\
\text { compliance with } \\
\text { nicotine patch }\end{array}$ & $\begin{array}{l}\text { self-reported } 7-\mathrm{d} P \mathrm{P}, \\
\text { validated by } \mathrm{CO} \leq 10 \\
\text { ppm and saliva } \\
\text { cotinine } \leq 15 \mathrm{ng} / \mathrm{ml} \text { at } \\
16 \& 26 \text { weeks }\end{array}$ & 236 & $\begin{array}{l}\text { 2 wks: } 222 \\
8 \text { wks: } 220 \\
16 \text { wks: } 213 \\
26 \text { wks: } 222\end{array}$ & $\begin{array}{l}\text { GEE models predicting } \\
7-d \text { PP } \\
\text { no specific control for } \\
\text { non-adherence due to } \\
\text { relapse }\end{array}$ & $\begin{array}{l}\text { OR of quitting for greater adher- } \\
\text { ence: } 2.23 ; \mathrm{p}<0.0001 \text { (no } 95 \% \mathrm{Cl} \\
\text { provided) }\end{array}$ \\
\hline [15] & $\begin{array}{l}\text { USA } \\
\text { 2001-2004 } \\
\text { smokers } \geq 10 / d\end{array}$ & $\begin{array}{l}46.1 \% \text { women } \\
86 \% \text { white }\end{array}$ & $\begin{array}{l}\text { RCT of extended BUP } \\
\text { vs. PLC following an } \\
\text { 11-wk programme } \\
\text { with BUP + NRT }\end{array}$ & $\begin{array}{l}\text { Last clinic visit at } \\
\text { week 25; tele- } \\
\text { phone follow-up } \\
\text { (IVR system) until } \\
52 \text { months }\end{array}$ & $\begin{array}{l}\text { Internet, } \\
\text { newspaper } \\
\text { \&radio ads, } \\
\text { local organiza- } \\
\text { tions (12/01- } \\
3 / 04)\end{array}$ & $\begin{array}{l}\text { positive answer to } \\
\text { the questions "Are } \\
\text { you wearing a patch } \\
\text { now" and "Have you } \\
\text { taken your pill this } \\
\text { morning?" } \\
\text { no clear definition of } \\
\text { adherence } \\
\end{array}$ & $\begin{array}{l}\text { self-reported PP, } \\
\text { validated by CO<10 } \\
\text { ppm at } 52 \text { weeks } \\
\text { (special appointment } \\
\text { for self-reported } \\
\text { non-smokers) }\end{array}$ & 362 & 362 & $\begin{array}{l}\text { logistic regression } \\
\text { no specific control for } \\
\text { non-adherence due to } \\
\text { relapse }\end{array}$ & $\begin{array}{l}\text { OR of quitting for more frequent } \\
\text { use of study medication (including } \\
\text { placebo) at... } \\
-25 \text { wks: } 1.7(1.5-2.0) \\
-52 \text { wks: } 1.61(1.4-1.9)\end{array}$ \\
\hline [16] & $\begin{array}{l}\text { USA } \\
\text { before } 2007 \\
\text { female smokers } \geq 10 / d\end{array}$ & $\begin{array}{l}47.8 \pm 9.3 \mathrm{yrs} \\
100 \% \text { women } \\
70.1 \% \text { white } \\
21.4 \pm 9.2 \mathrm{cig} / \mathrm{d} \\
\text { FTND } 5.8 \pm 2.3\end{array}$ & $\begin{array}{l}\text { RCT of BUP vs. PLC and } \\
\text { CBT vs. support }(2 \times 2 \\
\text { design) }\end{array}$ & $\begin{array}{l}12 \text { months, but } \\
\text { the association } \\
\text { between adher- } \\
\text { ence and absti- } \\
\text { nence was only } \\
\text { assessed at } 7 \\
\text { weeks (EOT) }\end{array}$ & $\begin{array}{l}\text { radio, televi- } \\
\text { sion and print } \\
\text { ads }\end{array}$ & $\begin{array}{l}\text { MEMS: adherence = } \\
\text { taking } 2 \text { doses } / \mathrm{d} \\
\text { Adherence score = } \\
\text { days of adherence / } \\
42 \text { days of } \mathrm{Tx}\end{array}$ & $\begin{array}{l}\text { self-reported 7-d PP, } \\
\text { validated by CO and } \\
\text { salivary cotinine }<15 \\
\mathrm{ng} / \mathrm{ml}\end{array}$ & 154 & $\begin{array}{l}\text { EOT: } 81 \\
3 \mathrm{mo}: 69 \\
6 \mathrm{mo}: 60 \\
9 \mathrm{mo}: 69 \\
12 \mathrm{mo}: 70\end{array}$ & $\begin{array}{l}\text { logistic regression for } \\
\text { predictors of abstinence } \\
\text { at } 7 \text { weeks } \\
\text { no specific control for } \\
\text { non-adherence due to } \\
\text { relapse }\end{array}$ & $\begin{array}{l}\text { For participants receiving BUP, } \\
\text { adherence } \\
\text { levels did not predict abstinence } \\
\text { rates at EOT }\end{array}$ \\
\hline [17] & $\begin{array}{l}\text { USA } \\
\text { 2001-2003 } \\
\text { smokers treated at a } \\
\text { tobacco dependence } \\
\text { clinic }\end{array}$ & $\begin{array}{l}44 \pm \text { ?? yrs } \\
61 \% \text { women } \\
71 \% \text { Caucas. } \\
22 \pm \text { ?? cig/d }\end{array}$ & $\begin{array}{l}\text { retrospective cohort } \\
\text { analysis of smokers } \\
\text { using } \geq 1 \text { form of NRT }\end{array}$ & $\begin{array}{l}\text { 4-wk data were } \\
\text { collected at visits } \\
\text { or by phone } \\
\text { 26-wk data were } \\
\text { collected by } \\
\text { phone or mail }\end{array}$ & not described & $\begin{array}{l}\text { no a priori definition } \\
\text { of adherence }\end{array}$ & $\begin{array}{l}\text { self-reported } 7-d P P \\
\text { at } 4 \text { and } 26 \text { weeks, } \\
\text { validated by } C O<10 \\
\text { ppm in a subsample } \\
(n=255) \text { at } 4 \text { weeks }\end{array}$ & 790 & $\begin{array}{l}26 \text { wks: } 626 \\
\text { (dropouts were } \\
\text { considered to be } \\
\text { smoking and not } \\
\text { using NRT) }\end{array}$ & $\begin{array}{l}\chi^{2} \text { test } \\
\text { no specific control for } \\
\text { non-adherence due to } \\
\text { relapse after } 4 \text { weeks }\end{array}$ & $\begin{array}{l}\text { 7-d PP at } 6 \text { months in those using } \\
\text { NRT for } \geq 6 \text { mo vs. }<6 \text { mo: } 65 \% \text { vs. } \\
27 \% ; \text {; }<0.001 \text { (subgroup of these } \\
\text { who were abstinent at } 4 \text { wss: } 82 \% \\
\text { vs. } 52 \% ; \text { p } 20.01 \text { ) }\end{array}$ \\
\hline [18] & $\begin{array}{l}\text { United Kingdom } \\
\text { before 1987 } \\
\text { smokers working in a } \\
\text { retailing company }\end{array}$ & $\begin{array}{l}34.3 \pm 10.6 \mathrm{yrs} \\
70 \% \text { women } \\
15.5 \pm 7.6 \mathrm{cig} / \mathrm{d}\end{array}$ & $\begin{array}{l}\text { quasi-randomised trial } \\
\text { of a 2-session cessa- } \\
\text { tion programme (of } \\
334 \text { interested } \\
\text { smokers, only } 270 \\
\text { were invited, and } 172 \\
\text { of these took part) } \\
\text { NRT had to be } \\
\text { purchased }\end{array}$ & 12 months & $\begin{array}{l}\text { mailing of } \\
\text { invitation } \\
\text { letters to } \\
\text { employees }\end{array}$ & $\begin{array}{l}\text { use of }>1 \text { box of } \\
\text { nicotine gum (105 } \\
\text { pieces) }\end{array}$ & $\begin{array}{l}\text { self-reported } \\
\text { continuous 12-month } \\
\text { abstinence (lenient } \\
\text { or strict definition), } \\
\text { validated by } \mathrm{CO}<10 \\
\mathrm{ppm}\end{array}$ & 334 & $\begin{array}{l}12 \mathrm{mo:} 331 \text { were } \\
\text { interviewed, but } \\
\text { only } 303 \text { in person }\end{array}$ & $\begin{array}{l}\text { descriptive analysis } \\
\text { no specific control for } \\
\text { non-adherence due to } \\
\text { relapse }\end{array}$ & $\begin{array}{l}\text { 12-month continuous abstinence } \\
\text { rates in those using >105 pieces of } \\
\text { gum vus. those using } \leq 105 \text { pieces: } \\
19 \% \text { vs. } 9 \% \text { (no p value given) }\end{array}$ \\
\hline [19] & $\begin{array}{l}\text { United Kingdom } \\
\text { before } 1988 \\
\text { smokers working in } \\
\text { four companies }\end{array}$ & $\begin{array}{l}40 \pm \text { ?? yrs } \\
43 \% \text { women } \\
19 \pm \text { ?? yrs }\end{array}$ & $\begin{array}{l}\text { quasi-randomised trial } \\
\text { of a 3-month cessation } \\
\text { programme } \\
\text { NRT had to be } \\
\text { purchased }\end{array}$ & 12 months & $\begin{array}{l}\text { participants of } \\
\text { an earlier trial } \\
\text { of videos to } \\
\text { support } \\
\text { quitting }\end{array}$ & $\begin{array}{l}\text { use of }>1 \text { box of } \\
\text { nicotine gum (105 } \\
\text { pieces) }\end{array}$ & $\begin{array}{l}\text { self-reported PP at } \\
12 \text { months, validated } \\
\text { by CO<10 ppm }\end{array}$ & $\begin{array}{l}161(79 \text { interv., } 82 \\
\text { control) of which } \\
32 \text { entered the } \\
\text { programme }\end{array}$ & 161 & $\begin{array}{l}\text { descriptive analysis } \\
\text { no specific control for } \\
\text { non-adherence due to } \\
\text { relapse }\end{array}$ & $\begin{array}{l}\text { no significant association between } \\
\text { amount of gum used and absti- } \\
\text { nence }\end{array}$ \\
\hline [20] & $\begin{array}{l}\text { USA } \\
\text { before } 2000 \\
\text { smokers } \geq 10 / d\end{array}$ & $\begin{array}{l}\text { mean age } \sim 46 \text { yrs } \\
\sim 46 \% \text { women } \\
\sim 87 \% \text { white } \\
\sim 25.5 \mathrm{cig} / \mathrm{d}\end{array}$ & $\begin{array}{l}\text { RCT of } 2 \text { different } \\
\text { doses of paroxetine or } \\
\text { PLC i iddition to } \\
\text { nicotine patches }\end{array}$ & $\begin{array}{l}4,10 \text { and } 26 \\
\text { weeks (interactive } \\
\text { voice response } \\
\text { system) and clinic } \\
\text { visits in those who } \\
\text { claimed to be } \\
\text { abstinent) }\end{array}$ & newspaper ad & $\begin{array}{l}\text { no a priori definition } \\
\text { of adherence }\end{array}$ & $\begin{array}{l}\text { self-reported } 7-\mathrm{d} \text { PP } \\
\text { at } 10 \& 26 \mathrm{wks}, \\
\text { validated by CO }<9 \\
\text { ppm and saliva } \\
\text { cotinine }<20 \mathrm{ng} / \mathrm{ml}\end{array}$ & 224 & 224 & $\begin{array}{l}\text { logistic regression for } \\
\text { the association between } \\
\text { the number of affirea- } \\
\text { tive statements on } \\
\text { adherence on the } \\
\text { telephone and absti- } \\
\text { nence at } 4 \text { weeks }\end{array}$ & $\begin{array}{l}\text { OR of abstinence at } 4 \text { weeks for } \\
\text { more affirmative statements: } 3.27 \\
(2.0-5.2)\end{array}$ \\
\hline [21] & $\begin{array}{l}\text { USA } \\
\text { before } 1992 \\
\text { smokers (50\% were } \\
\text { employees of the } \\
\text { hospital where the } \\
\text { study was conducted) } \\
\end{array}$ & $\begin{array}{l}38.9 \pm 8.9 \mathrm{yrs} \\
66.6 \% \text { women } \\
28.4 \pm 12.5 \mathrm{cig} / \mathrm{d} \\
\text { FTND } 6.6 \pm 1.7\end{array}$ & $\begin{array}{l}\text { observational study of } \\
\text { voluntary NRT use in a } \\
\text { 10-session group } \\
\text { cessation programme }\end{array}$ & 6 months & $\begin{array}{l}\text { hospital } \\
\text { publications }\end{array}$ & $\begin{array}{l}\text { subjects completed a } \\
\text { questionnaire on } \\
\text { gum use } 3 \text { wks after } \\
\text { TQD } \\
\text { adherence }=\text { used as } \\
\text { recommended }\end{array}$ & $\begin{array}{l}\text { self-reported PP at } 6 \\
\text { mo, validated by CO } \\
\text { (no cut-off provided) }\end{array}$ & 36 & 36 & $\begin{array}{l}\text { T tests, } \chi^{2} \text { tests } \\
\text { no specific control for } \\
\text { non-adherence due to } \\
\text { relapse }\end{array}$ & $\begin{array}{l}\text { abstinence rates in adherent vs. } \\
\text { non-adherent subjects: } 50 \% \text { vs. } 83 \% \\
\text { (n.s.) }\end{array}$ \\
\hline [22] & $\begin{array}{l}\text { USA } \\
\text { 2003-2004 } \\
\text { African-American light } \\
\text { smokers ( } \leq 10 / d)\end{array}$ & $\begin{array}{l}45.1 \pm 10.7 \mathrm{yrs} \\
66.9 \% \text { women } \\
7.6 \pm 3.2 \mathrm{cig} / \mathrm{d}\end{array}$ & $\begin{array}{l}\text { secondary analysis of } \\
\text { an RCT (name: 'KIS'; } \\
\text { full name not provid- } \\
\text { ed) of health educ. vs. } \\
\text { MI and nicotine gum } \\
\end{array}$ & $\begin{array}{l}\text { weeks } 1,3,6,8 \text {, } \\
\text { and } 26\end{array}$ & not reported & $\begin{array}{l}\text { use of } \geq 75 \% \text { of the } \\
\text { prescribed dose }\end{array}$ & $\begin{array}{l}\text { self-reported } 7 \text {-d PP } \\
\text { at } 26 \text { wks, validated } \\
\text { by saliva cotinine } \leq 20 \\
\mathrm{ng} / \mathrm{ml}\end{array}$ & $\begin{array}{l}755 \text { of which } 378 \\
\text { were randomised } \\
\text { to verum (gum) }\end{array}$ & $\begin{array}{l}\text { week 26: } 637 \\
662 \text { were included } \\
\text { in the adherence } \\
\text { analyses }\end{array}$ & $\begin{array}{l}\text { descriptive analyses and } \\
\text { multiple logistic } \\
\text { regression for predic- } \\
\text { tors of abstinence } \\
\text { no specific control for }\end{array}$ & $\begin{array}{l}\text { abstinence rates at } 26 \text { wks for } \\
\text { adherent vs. non-adherent subjects: } \\
9.5 \% \text { vs. } 16.7 \% \text { (no p value given) } \\
\text { adjusted OR of quitting for adher- }\end{array}$ \\
\hline
\end{tabular}




\begin{tabular}{|c|c|c|c|c|c|c|c|c|c|c|c|}
\hline & & & $\begin{array}{l}\text { vs. PLC ( } 2 \times 2 \text { design); } \\
\text { 3/03-6/04 }\end{array}$ & & & & & & & $\begin{array}{l}\text { non-adherence due to } \\
\text { relapse }\end{array}$ & $\begin{array}{l}\text { ence to study medication (including } \\
\text { placebo) at } 26 \text { wks: } 0.50(0.28-0.87)\end{array}$ \\
\hline [23] & $\begin{array}{l}\text { USA } \\
\text { before } 1995 \\
\text { smokers }\end{array}$ & $\begin{array}{l}42.2 \pm 9.7 \mathrm{yrs} \\
51 \% \text { women } \\
33.0 \pm 10.6 \mathrm{cig} / \mathrm{d} \\
\text { FTND } 7.8 \pm 1.5 \\
\end{array}$ & $\begin{array}{l}\text { RCT of } 4 \text { gum use } \\
\text { durations }(0 / 7 / 15 / \\
30 \text { pcs/d) in addition to } \\
\text { minimal support } \\
\end{array}$ & up to 24 weeks & $\begin{array}{l}\text { newspaper and } \\
\text { radio ads }\end{array}$ & $\begin{array}{l}\text { no a priori definition } \\
\text { of adherence; gum } \\
\text { use was assessed by } \\
\text { self-report }\end{array}$ & $C O \leq 10 \mathrm{ppm}$ & 177 & 177 & $\begin{array}{l}\text { ANOVA (4 groups) } \\
\text { no specific control for } \\
\text { non-adherence due to } \\
\text { relapse }\end{array}$ & $\begin{array}{l}\text { no overall effect of gum-group } \\
\text { assignment on abstinence }\end{array}$ \\
\hline [24] & $\begin{array}{l}\text { United Kingdom } \\
1980 \\
\text { smokers who were } \\
\text { offered NRT gum }\end{array}$ & $\begin{array}{l}\text { mean age: } 40 \text { yrs } \\
56 \% \text { women } \\
\text { mean cig/d: } 17.9\end{array}$ & $\begin{array}{l}\text { subgroup analysis of a } \\
\text { cessation intervention } \\
\text { trial }\end{array}$ & 4 and 12 months & not reported & $\begin{array}{l}\text { Categorical variable } \\
\text { 'gum use': } 0 / 1-14 \\
\text { pcs } / 15 \text { 15-105 pcs / } \\
>105 \text { pcs }\end{array}$ & $\begin{array}{l}\text { self-reported PP at } 4 \\
\& 12 \text { mo, validated } \\
\text { by } C \text { in a subsample } \\
\text { at } 12 \text { months }\end{array}$ & 679 & 474 & $\begin{array}{l}\text { logistic regression for } \\
\text { predictors of abstinence } \\
\text { no specific control for } \\
\text { non-adherence due to } \\
\text { relapse }\end{array}$ & $\begin{array}{l}\text { Use of }>105 \mathrm{pcs} / \mathrm{d} \text { was associated } \\
\text { with significantly higher } 4 \text {-mo and } \\
12 \text {-mo abstinence rates that use of } \\
\leq 105 \mathrm{pcs} / \mathrm{d} \text {. } \\
\text { ORs are not reported }\end{array}$ \\
\hline [25] & $\begin{array}{l}\text { USA } \\
\text { 1989-1990 } \\
\text { highly-dependent } \\
\text { smokers (FTND } \geq 7 \text { ) }\end{array}$ & $\begin{array}{l}\text { mean age } \sim 40 \text { yrs } \\
42.8 \% \text { women } \\
97 \% \text { Caucas. } \\
\text { mean FTND } \sim 8.4\end{array}$ & $\begin{array}{l}\text { RCT of } 2 \text { doses of } \\
\text { nicotine gum }(4 / 2 \mathrm{mg}) \\
\text { vs. PLC in addition to a } \\
\text { minimal intervention; } \\
5 / 89-5 / 90\end{array}$ & 6 weeks & not reported & $\begin{array}{l}\text { using an average of } \\
\geq 9 \mathrm{pcs} / \mathrm{d}\end{array}$ & $\begin{array}{l}\text { continuous 28-day } \\
\text { abstinence at week } \\
6 \text {, validated by } \\
\text { CO }<10 \mathrm{ppm}\end{array}$ & 563 & 216 & $\begin{array}{l}\text { 4-wk abstinence was } \\
\text { calculated separately } \\
\text { for adherent and non- } \\
\text { adherent pts. in the } \\
\text { three groups but were } \\
\text { only compared between } \\
\text { groups }\end{array}$ & $\begin{array}{l}\text { abstinence rates in adherent } \\
\text { subjects: } 56 \%(4 \mathrm{mg}) / 33 \%(2 \mathrm{mg}) / \\
33 \% \text { (PL); } p=0.0016 \text { (no sign. } \\
\text { difference in non-adherent } \\
\text { subjects) }\end{array}$ \\
\hline [26] & $\begin{array}{l}\text { USA } \\
\text { before 1999 } \\
\text { heavy smokers } \\
\text { (>25/d) }\end{array}$ & $\begin{array}{l}\text { mean age } \sim 47 \text { yrs } \\
41.4 \% \text { women } \\
\sim 82.5 \% \text { white } \\
\text { mean cig/d } \sim 36\end{array}$ & $\begin{array}{l}\text { RCT of } 25 \mathrm{mg} \text { vs. } 15 \mathrm{mg} \\
\text { nicotine patches (6 } \\
\text { weekss in addition to } \\
\text { self-help material }\end{array}$ & $\begin{array}{l}2 \mathrm{mo} \text { (clinic visit) } \\
6 \& 12 \text { months } \\
\text { (phone visit) }\end{array}$ & newspaper ads & $\begin{array}{l}\text { adherence was } \\
\text { assessed at } 1,2,4 \& \\
6 \text { wks (IVR system) } \\
\text { definition of adher- } \\
\text { ence: positive } \\
\text { answer to the } \\
\text { question "Are you } \\
\text { wearing a patch } \\
\text { now?" }\end{array}$ & $\begin{array}{l}\text { self-reported } 7-\mathrm{d} \mathrm{PP} \\
\text { at various time- } \\
\text { points, validated by } \\
\text { CO<9 ppm and saliva } \\
\text { cotinine }<20 \mathrm{ng} / \mathrm{ml} \text { at } \\
2 \text { months }\end{array}$ & 408 & $\begin{array}{l}\text { IVR calls: } \\
\text { wk 1: } \sim 380 \\
\text { wk 2: } \sim 370 \\
\text { wk } 4: \sim 530 \\
\text { wk 6: } \sim 290\end{array}$ & $\begin{array}{l}\chi^{2} \text { Test comparing } \\
\text { adherent and non- } \\
\text { adherent subjects } \\
\text { no specific control for } \\
\text { non-adherence due to } \\
\text { relapse }\end{array}$ & $\begin{array}{l}\text { validated abstinence rates at } 2 \\
\text { months in adherent vs. non- } \\
\text { adherent subjects: } 28 \% \text { vs. } 11 \% \\
\text { (p<0.001) }\end{array}$ \\
\hline [27] & $\begin{array}{l}\text { USA } \\
\text { before } 2007 \\
\text { female smokers } \geq 10 / d\end{array}$ & $\begin{array}{l}42.1 \pm 10.0 \text { yrs } \\
100 \% \text { women } \\
61.8 \% \text { Caucas. } \\
20.8 \pm 8.8 \text { cig/d } \\
\text { FTND } 5.0 \pm 2.5\end{array}$ & $\begin{array}{l}\text { RCT of using MEMS } \\
\text { feedback to increase } \\
\text { adherence to a } 7-w k \\
\text { course of BUP }\end{array}$ & 6 weeks & not reported & $\begin{array}{l}\text { MEMS: a) dose } \\
\text { adherence =taking } 2 \\
\text { doses per day; b) full } \\
\text { adherence }=2 \text { doses } \\
\text { per day, } 8-12 \text { hapart } \\
\text { The intervention } \\
\text { grour received an } \\
\text { intervention to } \\
\text { increase adherence! }\end{array}$ & $\begin{array}{l}\text { self-reported 7-d PP } \\
\text { at } 3 \& 6 \text { weeks, } \\
\text { validated by CO (no } \\
\text { specific information } \\
\text { provided) }\end{array}$ & $\begin{array}{l}55 \text { (intervention } \\
27 \text {, control 28) }\end{array}$ & 24 overall & $\begin{array}{l}\text { effect sizes expressed in } \\
\text { terms of multiple } \\
\text { regression coefficients } \\
\text { no specific control for } \\
\text { non-adherence due to } \\
\text { relapse }\end{array}$ & $\begin{array}{l}\text { At } 3 \& 6 \text { wks, both types of adher- } \\
\text { ence were associated with higher } \\
\text { abstinence rates (all } p<0.05 \text { ) }\end{array}$ \\
\hline [28] & $\begin{array}{l}\text { USA } \\
2009-2010 \\
\text { black smokers }>10 / d\end{array}$ & $\begin{array}{l}46.8 \pm 11.3 \mathrm{yrs} \\
62.5 \% \text { women } \\
100 \% \text { black } \\
16.3 \pm 5.4 \mathrm{cig} / \mathrm{d}\end{array}$ & $\begin{array}{l}\text { RCT of adherence } \\
\text { support vs. usual care } \\
\text { in addition to a 12-wk } \\
\text { course of VAR; } 3 / 09- \\
8 / 09\end{array}$ & 12 weeks & not reported & $\begin{array}{l}\text { adherence was } \\
\text { assessed by pill } \\
\text { counts and is } \\
\text { expressed as a } \\
\text { percentage }\end{array}$ & $\begin{array}{l}\text { self-reported } 7-\mathrm{d} P \mathrm{P} \\
\text { at } 12 \text { wks, validated } \\
\text { by saliva cotinine }<20 \\
\mathrm{ng} / \mathrm{ml}\end{array}$ & 72 & $\begin{array}{l}4 \text { weeks: } 60 \\
8 \text { weeks: } 57 \\
12 \text { weeks: } 61\end{array}$ & $\begin{array}{l}\chi^{2} \text { test } \\
\text { no specific control for } \\
\text { non-adherence due to } \\
\text { relapse }\end{array}$ & $\begin{array}{l}\text { adherence in quitters vs. relapsers } \\
\text { at } 12 \text { weeks: } 95.8 \% \text { vs. } 80.8 \% \text {; } \\
\text { p<0.05 }\end{array}$ \\
\hline [29] & $\begin{array}{l}\text { USA } \\
2004 \\
\text { homeless smokers }\end{array}$ & $\begin{array}{l}\text { mean age } \sim 44 \text { yrs } \\
\sim 39.1 \% \text { women } \\
\sim 30 \% \text { white } \\
\text { mean cig/d } \sim 15 \\
\text { mean FTND } \sim 4\end{array}$ & $\begin{array}{l}\text { RCT of two different } \\
\text { counselling formats in } \\
\text { addition to NRT (self- } \\
\text { selection of patch or } \\
\text { gum); } 2 / 04-12 / 04\end{array}$ & $\begin{array}{l}26 \text { weeks (associa- } \\
\text { tion between } \\
\text { adherence \& } \\
\text { abstinence was } \\
\text { only assessed at } 8 \\
\text { weeks) }\end{array}$ & $\begin{array}{l}\text { flyers distribut- } \\
\text { ed at } 13 \\
\text { homeless } \\
\text { service facilities }\end{array}$ & $\begin{array}{l}\text { adherence to patch: } \\
1 \text { patch/d } \\
\text { adherence to gum: } \\
\text { according to a } \\
\text { tailored dosing } \\
\text { schedule (no details } \\
\text { provided) }\end{array}$ & $\begin{array}{l}\text { self-reported } 7-d P P \\
\text { at } 26 \text { wks, validated } \\
\text { by } C O<10 \text { ppm and } \\
\text { saliva cotinine } \leq 20 \\
\mathrm{ng} / \mathrm{ml}\end{array}$ & 46 & 28 & $\begin{array}{l}\chi^{2} \text { test (presumably) } \\
\text { no specific control for } \\
\text { non-adherence due to } \\
\text { relapse }\end{array}$ & $\begin{array}{l}\text { abstinence rates at } 8 \text { wks for use of } \\
\geq 4 \mathrm{vs.}<4 \text { patches/wk: } 33.3 \% \text { vs. } \\
10.5 \% ; p=0.3 \text { (This information is } \\
\text { only given in the abstract, not in the } \\
\text { paper) }\end{array}$ \\
\hline [30] & $\begin{array}{l}\text { USA } \\
1989 \\
\text { highly-dependent } \\
\text { smokers (FTND } \geq 7 \text { ) }\end{array}$ & $\begin{array}{l}\text { mean age } \sim 43 \text { yrs } \\
44 \% \text { women } \\
\sim 91 \% \text { white }\end{array}$ & $\begin{array}{l}\text { RCT of } 3 \text { doses of } \\
\text { nicotine gum }(4 / 2 / \\
0.5 \mathrm{mg})\end{array}$ & $\begin{array}{l}1,2,4 \text { and } 6 \\
\text { weeks after TQD }\end{array}$ & newspaper ads & $\begin{array}{l}\text { pts. were instructed } \\
\text { to use } 12 \text { pcs/d and } \\
\text { to complete a usage } \\
\text { diary }\end{array}$ & $\begin{array}{l}\text { continuous } 28 \text {-day } \\
\text { abstinence at week } \\
6 \text {, validated by } C 0<8 \\
\text { ppm }\end{array}$ & 90 & 90 & $\begin{array}{l}\text { Mann-Whitney test } \\
\text { no specific control for } \\
\text { non-adherence due to } \\
\text { relapse }\end{array}$ & $\begin{array}{l}\text { At } 6 \text { weeks, abstinent subjects had } \\
\text { used significantly more gum than } \\
\text { relapsers }(10.9 \pm 2.1 \text { vs. } 9.7 \pm 1.6 \\
\text { pcs/d; p<0.03); this effect was } \\
\text { driven by a significant finding in the } \\
4 \mathrm{mg} \text { group }\end{array}$ \\
\hline [31] & $\begin{array}{l}\text { Denmark } \\
\text { before } 1993 \\
\text { smokers } \geq 10 / d\end{array}$ & $\begin{array}{l}\text { mean age } \sim 39 \text { yrs } \\
\sim 60 \% \text { women } \\
\text { mean cig/d } \sim 20 \\
\text { mean FTND } \sim 7.3\end{array}$ & $\begin{array}{l}\text { RCT of nicotine inhaler } \\
\text { vs. PLC }\end{array}$ & $\begin{array}{l}12 \text { months } \\
\text { (association } \\
\text { between adher- } \\
\text { ence \& abstinence } \\
\text { was only assessed } \\
\text { at } 6 \text { weeks) }\end{array}$ & newspaper ads & $\begin{array}{l}\text { pts. were instructed } \\
\text { to use } 2-10 \text { inhal- } \\
\text { ers/d and to com- } \\
\text { plete a usage diary } \\
\text { for the first } 3 \text { wks of } \\
\text { the trial }\end{array}$ & $\begin{array}{l}\text { self-reported } \\
\text { continuous absti- } \\
\text { nence at } 12 \text { months, } \\
\text { validated by } \mathrm{CO}<10 \\
\text { ppm }\end{array}$ & 286 & 12 months: 273 & $\begin{array}{l}\text { logistic regression for } \\
\text { predictors of abstinence } \\
\text { at } 6 \text { weeks } \\
\text { no specific control for } \\
\text { non-adherence due to } \\
\text { relapse }\end{array}$ & $\begin{array}{l}\text { In the active group, abstinent } \\
\text { subjects at week } 6 \text { had used more } \\
\text { inhalers/d than relapsers ( } p=0.008 \text { ) } \\
\text { OR of abstinence at } 6 \text { weeks for } \\
\text { number of inhalers used: } 1.41 \text { (no } \\
\text { CI provided) }\end{array}$ \\
\hline
\end{tabular}




\begin{tabular}{|c|c|c|c|c|c|c|c|c|c|c|c|}
\hline [32] & $\begin{array}{l}\text { Malaysia } \\
2009-2010 \\
\text { smokers working at } \\
\text { two public universities }\end{array}$ & $\begin{array}{l}35.9 \pm 10.9 \text { yrs } \\
0 \% \text { women }\end{array}$ & $\begin{array}{l}\text { observational study of } \\
\text { a behavioural interven- } \\
\text { tion and free NRT; } \\
11 / 09-6 / 10\end{array}$ & 8 weeks & $\begin{array}{l}\text { invitation letter } \\
\text { and e-mail, } \\
\text { Health screen- } \\
\text { ings, 'Wellness } \\
\text { Day' }\end{array}$ & NRT use for $\geq 2$ weeks & $\begin{array}{l}\text { validated by } \mathrm{CO}<10 \\
\mathrm{ppm}\end{array}$ & 185 & not reported & $\begin{array}{l}\text { logistic regression for } \\
\text { predictors of abstinence } \\
\text { at } 2 \text { months } \\
\text { no specific control for } \\
\text { non-adherence due to } \\
\text { relapse }\end{array}$ & $\begin{array}{l}\text { According to the abstract, adher- } \\
\text { ence to NRT was a univariate } \\
\text { predictor of cessation }(p<0.001) \text {. } \\
\text { Apparently, adherence was not } \\
\text { entered in the multivariate model. }\end{array}$ \\
\hline [33] & $\begin{array}{l}\text { USA } \\
2005-2008 \\
\text { smokers } \geq 10 \text { cig/d } \\
\text { with attention-deficit/ } \\
\text { hyperactivity disorder }\end{array}$ & $\begin{array}{l}37.8 \pm 10.0 \mathrm{yrs} \\
43.5 \% \text { women } \\
82.6 \% \text { white } \\
19.9 \pm 7.7 \mathrm{cig} / \mathrm{d} \\
\text { FTND } 5.5 \pm 2.2\end{array}$ & $\begin{array}{l}\text { secondary analysis of } \\
\text { an RCT of } \\
\text { methylphenidate or } \\
\text { PLC in addition to } \\
\text { nicotine patches and } \\
\text { counselling; } 12 / 05- \\
1 / 08\end{array}$ & 10 weeks & $\begin{array}{l}\text { advertising, } \\
\text { letters to clinic } \\
\text { patients, } \\
\text { networking } \\
\text { with communi- } \\
\text { ty professionals }\end{array}$ & $\begin{array}{l}\text { self-reported patch } \\
\text { adherence: number } \\
\text { of patches used } \\
\text { divided by the } \\
\text { number dispensed }\end{array}$ & $\begin{array}{l}\text { a) prolonged } \\
\text { abstinence during } \\
\text { weeks } 7-10 \text { (i.e. not } \\
\text { smoking on } 7 \\
\text { consecutive days or } \\
\text { at least once per } \\
\text { week for } 2 \text { consecu- } \\
\text { tive weeks) } \\
\text { b) self-reported } \\
\text { continuous absti- } \\
\text { nence weeks } 7 \\
\text { through 10, validated } \\
\text { by CO (no cut-off } \\
\text { provided) } \\
\text { c) self-reported 7-d } \\
\text { PP at } 10 \text { weeks }\end{array}$ & 255 & not reported & $\begin{array}{l}\text { mediation model to } \\
\text { assess relationships } \\
\text { between thoughts } \\
\text { about abstinence } \\
\text { (predictors), adherence } \\
\text { (mediator) and absti- } \\
\text { nence (outcome); } \\
\text { bootstrapped logistic } \\
\text { regression } \\
\text { no specific control for } \\
\text { non-adherence due to } \\
\text { relapse }\end{array}$ & $\begin{array}{l}\text { When factoring out predictor } \\
\text { variables, the mediator variable } \\
\text { 'patch adherence' was positively } \\
\text { associated with all three outcomes } \\
\text { (regression coefficients around } 0.3 \text { ). }\end{array}$ \\
\hline [34] & $\begin{array}{l}\text { Korea } \\
2007-2009 \\
\text { smokers attending a } \\
\text { smoking cessation } \\
\text { clinic }\end{array}$ & $\begin{array}{l}\text { mean age } \sim 48 \text { yrs } \\
0 \% \text { women } \\
\text { median cig/d: } 20 \\
\text { median FTND: } 5\end{array}$ & $\begin{array}{l}\text { retrospective analysis } \\
\text { of smokers receiving } \\
\text { VAR as part of a } \\
\text { cessation programme } \\
(9 / 07-12 / 09)\end{array}$ & 6 months & not reported & $\begin{array}{l}\text { no a priori definition } \\
\text { of adherence; no } \\
\text { description of } \\
\text { adherence meas- } \\
\text { urement (presuma- } \\
\text { bly self-report) } \\
\end{array}$ & $\begin{array}{l}\text { self-reported 6- } \\
\text { month continuous } \\
\text { rate, validated by CO } \\
\text { (no cut-off provided) }\end{array}$ & 87 & 78 & $\begin{array}{l}\text { logistic regression for } \\
\text { predictors of 6-month } \\
\text { continuous abstinence } \\
\text { no specific control for } \\
\text { non-adherence due to } \\
\text { relapse }\end{array}$ & $\begin{array}{l}\text { unadjusted OR of abstinence per } \\
\text { additional week of medication use: } \\
1.123 \text { (1.032-1.222) } \\
\text { adjusted OR: } 1.172 \text { (1.052-1.305) }\end{array}$ \\
\hline [35] & $\begin{array}{l}\text { USA } \\
\text { before } 2004 \\
\text { smokers } \geq 10 \mathrm{cig} / \mathrm{d}\end{array}$ & $43.3 \%$ women & $\begin{array}{l}\text { RCT of three intensities } \\
\text { of cognitive- } \\
\text { behavioural support in } \\
\text { addition to free } \\
\text { nicotine patches }\end{array}$ & $\begin{array}{l}7 \text { weeks, } \\
6 \text { months, } \\
12 \text { months }\end{array}$ & $\begin{array}{l}\text { subjects were } \\
\text { directly } \\
\text { approached by } \\
\text { primary care } \\
\text { providers }\end{array}$ & $\begin{array}{l}\text { three levels of } \\
\text { adherence (self- } \\
\text { report at } 7 \text { weeess): } \\
\text { - full: using all } \\
\text { patches } \\
\text { - partial: using most } \\
\text { or some patches } \\
\text { - none: using a bit or } \\
\text { none of the patches } \\
\end{array}$ & $\begin{array}{l}\text { self-reported } 7-d \text { PP } \\
\text { at } 7 \text { wks, } 6 \text { months } \\
\text { and } 12 \text { months, } \\
\text { validated by CO }<10 \\
\text { ppm }\end{array}$ & 619 & $\begin{array}{l}7 \text { weeks: } 485 \\
6 \text { months: not } \\
\text { reported } \\
12 \text { months: not } \\
\text { reported }\end{array}$ & $\begin{array}{l}\text { logistic regression for } \\
\text { predictors of abstinence } \\
\text { no specific control for } \\
\text { non-adherence due to } \\
\text { relapse }\end{array}$ & $\begin{array}{l}\text { OR of abstinence in fully adherent } \\
\text { (vs. all other groups) subjects: } \\
-7 \text { weeks: } 1.71(1.14-2.58) \\
-6 \text { months: } 2.47(1.56-3.91) \\
-12 \text { months: } 2.12(1.34-3.37)\end{array}$ \\
\hline [36] & $\begin{array}{l}\text { USA } \\
\text { before } 1997 \\
\text { smokers }\end{array}$ & $\begin{array}{l}\text { mean age } \sim 44 \text { yrs } \\
49.5 \% \text { women } \\
\sim 82 \% \text { white } \\
\text { mean cig/d } \sim 23\end{array}$ & $\begin{array}{l}\text { secondary analysis of } \\
\text { an RCT }(2 \times 2 \text { factorial } \\
\text { design) of nicotine } \\
\text { patch }(21 \mathrm{mg}) \text { vs. PLC } \\
\text { and self-help with vs. } \\
\text { without video }\end{array}$ & $\begin{array}{l}2,6 \text { and } 12 \\
\text { months }\end{array}$ & newspaper ads & $\begin{array}{l}\text { current patch use } \\
\text { was assessed via } \\
\text { telephone at } 24 \text { hrs, } \\
1 \text { week, } 2 \text { month hand } \\
2 \text { months. Full } \\
\text { compliance was } \\
\text { defined as asswering } \\
\text { 'yes' at all assess- } \\
\text { ments }\end{array}$ & $\begin{array}{l}\text { self-reported } 7-\mathrm{d} P P \\
\text { at } 2,6 \text { and } 12 \\
\text { months, validated by } \\
\mathrm{CO}<9 \mathrm{ppm} \text { and saliva } \\
\text { cotinine }<20 \mathrm{ng} / \mathrm{ml}\end{array}$ & 424 & $\begin{array}{l}6 \text { months: } 410 \\
12 \text { months: } 410\end{array}$ & $\begin{array}{l}\text { Cox proportional hazard } \\
\text { analysis of time to } \\
\text { relapse with compliance } \\
\text { status entered as an } \\
\text { independent variabe }\end{array}$ & $\begin{array}{l}\text { Patch compliance status entered } \\
\text { the model at } 2 \text { months }(p<0.001), 6 \\
\text { months }(p<0.001) \text { and } 12 \text { months } \\
(p<0.001) \text {. }\end{array}$ \\
\hline [37] & $\begin{array}{l}\text { United Kingdom } \\
2001-2003 \\
\text { smokers attending } \\
\text { Stop Smoking Services }\end{array}$ & $\begin{array}{l}44 \pm 12.7 \mathrm{yrs} \\
57 \% \text { women } \\
91 \% \text { white } \\
22.2 \pm 9.5 \mathrm{cig} / \mathrm{d} \\
\text { FTND } 5.8 \pm 2.1 \\
\end{array}$ & $\begin{array}{l}\text { prospective observa- } \\
\text { tional study including } \\
\text { smokers setting a quit } \\
\text { date and using BUP; } \\
1 / 01-12 / 03 \\
\end{array}$ & 4 weeks & not reported & $\begin{array}{l}\text { no a priori definition } \\
\text { of adherence; BUP } \\
\text { use was assessed by } \\
\text { self-report }\end{array}$ & $\begin{array}{l}\text { self-reported 14-d PP } \\
\text { four weeks after the } \\
\text { quit date, validated } \\
\text { by CO <10 ppm }\end{array}$ & 388 & 388 & $\begin{array}{l}\chi^{2} \text { test } \\
\text { no specific control for } \\
\text { non-adherence due to } \\
\text { relapse }\end{array}$ & $\begin{array}{l}\text { 14-d PP at four weeks depending on } \\
\text { BUP use in the week prior to the } \\
\text { quit date: } 44 \% \text { ( } \geq 14 \text { tablets) vs. } 32 \% \\
\text { ( }<14 \text { tablets); } P=0.26\end{array}$ \\
\hline
\end{tabular}

Table S1: Characteristics of studies which were excluded due to a lack of control for confounding by non-adherence due to relapse 


\section{References}

1. Alterman, A.I., P. Gariti, T.G. Cook, and A. Cnaan. Nicodermal patch adherence and its correlates. Drug Alcohol Depend, $1999 ; 53: 159-65$.

2. Balmford, J., R. Borland, D. Hammond, and K.M. Cummings. Adherence to and reasons for premature discontinuation from stop-smoking medications: data from the ITC Four-Country Survey. Nicotine Tob Res, 2011; 13: 94-102.

3. Catz, S.L., L.M. Jack, J.B. McClure, H.S. Javitz, M. Deprey, S.M. Zbikowski, T. McAfee, J. Richards, and G.E. Swan. Adherence to varenicline in the COMPASS smoking cessation intervention trial. Nicotine Tob Res, 2011; 13: 361-8.

4. Fucito, L.M., B.A. Toll, P. Salovey, and S.S. O'Malley. Beliefs and attitudes about bupropion: implications for medication adherence and smoking cessation treatment. Psychol Addict Behav, 2009; 23: 373-9.

5. Hays, J.T., S.J. Leischow, D. Lawrence, and T.C. Lee. Adherence to treatment for tobacco dependence: association with smoking abstinence and predictors of adherence. Nicotine Tob Res, 2010; 12: 574-81.

6. Lam, T.H., A.S. Abdullah, S.S. Chan, and A.J. Hedley. Adherence to nicotine replacement therapy versus quitting smoking among Chinese smokers: a preliminary investigation. Psychopharmacology (Berl), 2005; 177: 400-8.

7. Orleans, C.T., N. Resch, E. Noll, M.K. Keintz, B.K. Rimer, T.V. Brown, and T.M. Snedden. Use of transdermal nicotine in a state-level prescription plan for the elderly. A first look at 'real-world' patch users. JAMA, 1994; 271: 601-7.

8. Blak, B.T., K. Wilson, M. Metcalfe, A. Maguire, and M. Hards. Evaluation of varenicline as an aid to smoking cessation in UK general practice - a THIN database study. Curr Med Res Opin, 2010; 26: 861-70.

9. Shiffman, S., C.T. Sweeney, S.G. Ferguson, M.A. Sembower, and J.G. Gitchell. Relationship between adherence to daily nicotine patch use and treatment efficacy: secondary analysis of a 10-week randomized, double-blind, placebo-controlled clinical trial simulating over-the-counter use in adult smokers. Clin Ther, 2008; 30 : $1852-8$.

10. Cummings, K.M., R.M. Biernbaum, M.A. Zevon, T. Deloughry, and C.R. Jaen. Use and effectiveness of transdermal nicotine in primary care settings. Arch Fam Med, 1994; 3: 682-9.

11. Fagerstrom, K.O. Effects of nicotine chewing gum and follow-up appointments in physician-based smoking cessation. Prev Med, 1984 ; 13 : 517-27.

12. Goldstein, M.G., R. Niaura, M.J. Follick, and D.B. Abrams. Effects of behavioral skills training and schedule of nicotine gum administration on smoking cessation. Am J Psychiatry, 1989; 146: 56-60.

13. Grassi, M.C., D. Enea, A.K. Ferketich, B. Lu, S. Pasquariello, and P. Nencini. Effectiveness of varenicline for smoking cessation: a 1-year follow-up study. J Subst Abuse Treat, 2011; 41: 64-70.

14. Kahler, C.W., N.S. Spillane, J. Metrik, A.M. Leventhal, and P.M. Monti. Sensation seeking as a predictor of treatment compliance and smoking cessation treatment outcomes in heavy social drinkers. Pharmacol Biochem Behav, 2009; 93: 285-90.

15. Killen, J.D., S.P. Fortmann, G.M. Murphy, Jr., C. Hayward, C. Arredondo, D. Cromp, M. Celio, L. Abe, Y. Wang, and A.F. Schatzberg. Extended treatment with bupropion SR for cigarette smoking cessation. J Consult Clin Psychol, 2006; 74: 286-94.

16. Schmitz, J.M., A.L. Stotts, M.E. Mooney, K.A. Delaune, and G.F. Moeller. Bupropion and cognitive-behavioral therapy for smoking cessation in women. Nicotine Tob Res, 2007; 9: 699-709.

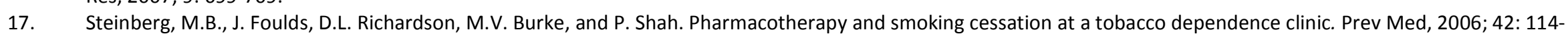
9.

18. Sutton, S. and R. Hallett. Randomized trial of brief individual treatment for smoking using nicotine chewing gum in a workplace setting. Am J Public Health, 1987; 77: 1210-1. 
19. Sutton, S. and R. Hallett. Smoking intervention in the workplace using videotapes and nicotine chewing gum. Prev Med, 1988; 17: 48-59.

20. Killen, J.D., S.P. Fortmann, A.F. Schatzberg, C. Hayward, L. Sussman, M. Rothman, L. Strausberg, and A. Varady. Nicotine patch and paroxetine for smoking cessation. J Consult Clin Psychol, 2000; 68: 883-9.

21. Millard, R.W., H.R. Waranch, and M. McEntee. Compliance to nicotine gum recommendations in a multicomponent group smoking cessation program: an exploratory study. Addict Behav, 1992; 17: 201-7.

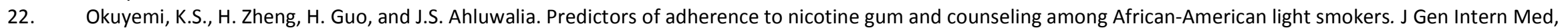
2010; 25: 969-76.

23. Gross, J., J. Johnson, L. Sigler, and M.L. Stitzer. Dose effects of nicotine gum. Addict Behav, 1995; $20: 371-81$.

24. Jackson, P.H., J.A. Stapleton, M.A. Russell, and R.J. Merriman. Nicotine gum use and outcome in a general practitioner intervention against smoking. Addict Behav, 1989; 14: 335-41.

25. Glover, E.D., D.P. Sachs, M.L. Stitzer, S.I. Rennard, W.C. Wadland, O.F. Pomerleau, R.T. Nowak, D.M. Daughton, P.N. Glover, J.R. Hughes, and J. Gross. Smoking cessation in highly dependent smokers with 4mg nicotine polacrilex. . Am J Health Behav, 1996; 20: 319-332.

26. Killen, J.D., S.P. Fortmann, L. Davis, L. Strausberg, and A. Varady. Do heavy smokers benefit from higher dose nicotine patch therapy? Exp Clin Psychopharmacol, 1999; 7: 226-33.

27. Mooney, M.E., S.L. Sayre, P.S. Hokanson, A.L. Stotts, and J.M. Schmitz. Adding MEMS feedback to behavioral smoking cessation therapy increases compliance with bupropion: a replication and extension study. Addict Behav, 2007; 32: 875-80.

28. Nollen, N.L., L.S. Cox, N. Nazir, E.F. Ellerbeck, A. Owen, S. Pankey, N. Thompson, and J.S. Ahluwalia. A pilot clinical trial of varenicline for smoking cessation in black smokers. Nicotine Tob Res, 2011; 13: 868-73.

29. Okuyemi, K.S., J.L. Thomas, S. Hall, N.L. Nollen, K.P. Richter, S.K. Jeffries, A.R. Caldwell, and J.S. Ahluwalia. Smoking cessation in homeless populations: a pilot clinical trial. Nicotine Tob Res, 2006; 8: 689-99.

Sachs, D.P. Effectiveness of the 4-mg dose of nicotine polacrilex for the initial treatment of high-dependent smokers. Arch Intern Med, 1995; 155: 1973-80.

Tonnesen, P., J. Norregaard, K. Mikkelsen, S. Jorgensen, and F. Nilsson. A double-blind trial of a nicotine inhaler for smoking cessation. JAMA, $1993 ; 269: 1268-71$.

Yasin, S.M., R. Masilamani, M.F. Ming, and D. Koh. Predictors of smoking cessation among staff in public Universities in Klang Valley, Malaysia. Asian Pac J Cancer Prev, 2011; 12: 811-6.

33. Heffner, J.L., D.F. Lewis, and T.M. Winhusen. Preliminary evidence that adherence to counseling mediates the effects of pretreatment self-efficacy and motivation on outcome of a cessation attempt in smokers with ADHD. Nicotine Tob Res, 2013; 15: 393-400.

34. Lee, J.Y., M.J. Kim, H.J. Jun, M. Kang, A.R. Park, D.E. Oh, Y.H. Choi, and J.H. Hwang. Adherence to varenicline and abstinence rates for quitting smoking in a private health promotion center-based smoking cessation clinic. Tuberc Respir Dis (Seoul), 2012; 72: 426-32.

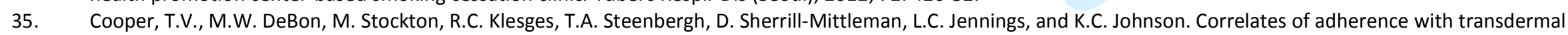
nicotine. Addict Behav, 2004; 29: 1565-78.

36. Killen, J.D., S.P. Fortmann, L. Davis, and A. Varady. Nicotine patch and self-help video for cigarette smoking cessation. J Consult Clin Psychol, $1997 ; 65$ : 663-72.

37. McEwen, A. and R. West. Do implementation issues influence the effectiveness of medications? The case of nicotine replacement therapy and bupropion in UK Stop Smoking Services. BMC Public Health, 2009; 9: 28. 


\section{$\underline{\text { Appendix }}$}

\section{SYSTEMATIC REVIEW PROTOCOL}

\section{A systematic review of studies assessing the association between}

\section{$\underline{\text { adherence to smoking cessation medication and treatment success }}$}

A number of studies suggest an association between the dose of cessation medication (i.e., daily dose or duration of use) and abstinence from smoking. There are two possible explanations for this finding: (1) Adherence to dosing regimens could be causally related to higher abstinence rates (i.e., non-adherence is a risk factor for failing to quit). (2) Patients who relapse may stop taking their medication. Thus, relapse during the study period could precipitate non-adherence in which case a reverse causality must be assumed (i.e., relapse precipitates non-adherence). Studies aimed at demonstrating that continuous abstinence is causally linked to medication adherence need to control for confounding by reverse causality. This can be done by either excluding all participants who relapsed before stopping their medication or by assessing adherence during a pre-specified treatment period and determine abstinence only in those subjects who had been continuously abstinent throughout this period.

\section{Review Questions}

Is there an association between adherence to cessation medication and continuous abstinence from smoking if reverse causality is being controlled for?

\section{Search terms}

Smoking cessation AND (adherence OR compliance) AND (abstinence OR success); (nicotine replacement OR bupropion OR varenicline) AND (adherence OR compliance)

The search terms are deliberately inclusive so that papers are not missed. 
Databases searched

Pubmed, WebOfScience, the Cochrane Tobacco Addiction Group specialized register

Hand search

Reference lists of included studies

Researchers contacted for knowledge of unpublished data/ongoing studies

- Professor Jonathan Foulds

Study Selection Criteria

Inclusion criteria

- General population sample (i.e. not recruited for particular clinical conditions)

- Adult participants ( $\geq 18$ years of age)

- First-line treatments (nicotine replacement therapy, bupropion, varenicline) alone or in combination

- Prospective design

- Specifically examining the association between medication adherence and continuous abstinence from smoking

- Published in peer-reviewed journals

- Written in English

- $\quad$ specific analysis controlling for reverse causality

Exclusion criteria

- Retrospective surveys (risk of confounding by recall bias)

- Review articles

- personal communications to editors

- commentaries

- study protocols

- case studies

- studies on smoking reduction

- studies involving pregnant women and adolescents

Search procedure

The lead reviewer will select studies for inclusion in the review. A second reviewer will independently screen all papers for suitability (using the study eligibility for review form).

Data to be extracted

- Study design

- Study sample and selection

- Outcome definition and measures

- Recall period

- Response rate

- Analysis

Data extraction strategy

Details of the studies agreed to be eligible for the review will be extracted and compiled into tables by the lead researcher and double-checked. All details in the table will be examined by a second reviewer highlighting any errors in extraction or 
discrepancies in interpretation between the reviewers. Any discrepancies will be discussed and resolved with the opinion of the other reviewers where necessary. 


\section{Study Eligibility for Review Form}

\section{General Information}

Pubmed ID:

Study Title:

Author contact details:

Identification number in the systematic review:

Identification of reviewer:

\section{Study Characteristics}

Verification of study eligibility

1) General population sample

2) Adult participants (e.g. $\geq 18$ years)

3) First-line treatments (NRT, bupropion, varenicline)

4) Prospective design

5) Specifically examining the association between medication adherence and continuous abstinence from smoking

6) Written in English

Notes: 
Table 1: Characteristics of studies examining the association between medication adherence and quitting success

\begin{tabular}{|c|c|c|c|c|c|c|c|c|c|c|c|}
\hline \multirow{2}{*}{ ID } & \multirow{2}{*}{$\begin{array}{l}\text { Country, Year } \\
\text { and Study } \\
\text { Population }\end{array}$} & \multirow{2}{*}{$\begin{array}{c}\text { Participant } \\
\text { characteristics }\end{array}$} & \multirow{2}{*}{$\begin{array}{l}\text { Design \& data } \\
\text { collection }\end{array}$} & \multirow{2}{*}{$\begin{array}{l}\text { Recall period } \\
\text { / length of } \\
\text { follow-up }\end{array}$} & \multirow{2}{*}{$\begin{array}{l}\text { Methods of } \\
\text { recruitment }\end{array}$} & \multicolumn{2}{|c|}{ Definitions } & \multicolumn{2}{|c|}{ Sample size } & \multirow{2}{*}{$\begin{array}{l}\text { Analysis method } \\
\text { (incl. control of } \\
\text { confounders) }\end{array}$} & \multirow{2}{*}{$\begin{array}{l}\text { Main findings regarding } \\
\text { the association between } \\
\text { adherence and abstinence }\end{array}$} \\
\hline & & & & & & adherence & success & Baseline & Follow-up & & \\
\hline & & & & & & & & & & & \\
\hline & & & & & & & & & & & \\
\hline & & & & 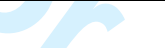 & $\Delta$ & & & & & & \\
\hline & & & & & & & & & & & \\
\hline & & & & & 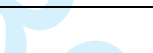 & & & & & & \\
\hline & & & & & $\sqrt{x}$ & & & & & & \\
\hline & & & & & 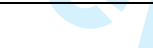 & $P=$ & & & & & \\
\hline & & & & & & 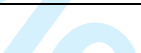 & & & & & \\
\hline & & & & & & $\sqrt{x}$ & & & & & \\
\hline & & & & & & & & & & & \\
\hline & & & & & & & & & & & \\
\hline & & & & & & & & & & & \\
\hline & & & & & & & & 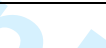 & & & \\
\hline & & & & & & & & & & & \\
\hline & & & & & & & & 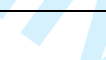 & & & \\
\hline & & & & & & & & $y$ & & & \\
\hline & & & & & & & & & & & \\
\hline
\end{tabular}




\section{Tables of outcome}

Data will be extracted and entered into the table by the lead reviewer and also independently by a second reviewer. Any discrepancies will be recorded, discussed and resolved.

Table 1: Studies examining the association between adherence and continuous abstinence and controlling for reverse causation.

Table 2: Studies examining the association between adherence and continuous abstinence without controlling for reverse causation.

\section{NOTES:}

- If more than one definition of success is examined (e.g. 1 week abstinence and 6 months abstinence) the longest length of abstinence that was linked to adherence data will be included in the study (i.e. 6 months).

- Where the association has been examined over multiple countries, the combined data-set will be used where available in preference to those that examine the association within each country individually. 


\section{$\underline{\text { Systematic review - second reviewer guide }}$}

1. Search through the 483 titles and abstracts and extract those that are eligible for the study. Please also note the reference of the studies that you required a fulltext document to ascertain if eligible. Please use the following codes to record the reasons for exclusion:

\begin{tabular}{|l|l|}
\hline 1 & not written in English \\
\hline 2 & no original data - review article \\
\hline 3 & $\begin{array}{l}\text { no original data - personal communications, commentaries, case reports, } \\
\text { study protocols, replies to other articles or "patient pages" }\end{array}$ \\
\hline 4 & unrelated to smoking cessation (but other conditions or smoking reduction) \\
\hline 5 & unrelated to pharmacotherapy \\
\hline 6 & unrelated to adherence \\
\hline 7 & including adolescents or pregnant women \\
\hline 8 & $\begin{array}{l}\text { other (e.g., no specific research question related to the association between } \\
\text { adherence and quitting success / predictors of adherence) }\end{array}$ \\
\hline 10 & no control for reverse causation \\
\hline
\end{tabular}

2. Check that you agree with all details entered in the Table (i.e. summary of included studies). Alter using track changes 1) any incorrect details, 2) details not needed, and 3) add anything missed that might be relevant

- Note any factors which you feel would be important to include in quality assessment of the studies.

Thank you! 